\title{
The Two-Dimensional Gaussian Beam Synthetic Method: Testing and Application
}

\begin{abstract}
R. NowaCK AND K. AKI
Department of Earth, Atmospheric, and Planetary Sciences, Massachusetts Institute of Technology, Cambridge

The Gaussian beam method of Cervený et al. (1982) is an asymptotic method for the computation of wave fields in inhomogeneous media. The method consists of tracing rays and then solving the wave equation in "ray-centered coordinates." The parabolic approximation is applied to find the asymptotic local solution in the neighborhod of each ray. The approximate global solution for a given source is then constructed by a superposition of Gaussian beams along nearby rays. The Gaussian beam method is tested in a two-dimensional inhomogeneous medium using two approaches. One is the application of the reciprocal theorem for Green's functions in an arbitrarily heterogeneous medium. The discrepancy between synthetic seismograms for reciprocal cases is considered as a measure of the error. The other approach is to apply Gaussian beam synthesis to cases for which solutions are known by other approximate methods. This includes the soft basin problem that has been studied by finite difference, finite element, discrete wavenumber, and glorified optics. We found that the results of these tests were in general satisfactory. We have used the Gaussian beam method for two applications. First, the method is used to study volcanic earthquakes at Mount Saint Helens. The observed large differences in amplitude and arrival time between a station inside the crater and stations on the flanks can be explained by the combined effects of an anomalous velocity structure and a shallow focal depth. The method is also applied to scattering of teleseismic $\boldsymbol{P}$ waves by a lithosphere with randomly fluctuating velocities.
\end{abstract}

\section{INTRODUCTION}

In this paper we will discuss applications of the Gaussian beam method. The Gaussian beam method is a variation of both the asymptotic ray method [ $\breve{C}$ erveny $\dot{e}$ et al., 1977] and the parabolic approximation method [Tappert, 1976; Claerbout, 1976]. The Gaussian beam method has been recently deScribed in the literature by Babich and Popov [1981], Popov [1981, 1982], and Červený et al. [1982]. These studies were based on the scalar wave equation. The elastic case was first derived by Kirpichnikova [1971] and more recently by Červený and Pšenčlk [1983b] for the two-dimensional case and by Červený and Pšenčik [1983a] for the three-dimensional case.

There are three basic steps involved in the Gaussian beam method. First, a system of rays must be traced from the source. Since high-frequency energy flows along rays, the system of rays can be thought of as the framework upon which the wave field is to be built. This step requires the numerical solution of the kinematic ray tracing system. Second, the wave equation (elastodynamic equation) is solved in "ray centered" curvilinear coordinates for each ray using the parabolic approximation. This is now a local parabolic approximation related to a particular ray trajectory. These local solutions can be expressed in terms of Gaussian beams. The final solution is the result of superposing local solutions along individual rays to give an approximate global solution for a given source condition. This step is justified by the linearity of the underlying wave equation.

There are several advantages in using the Gaussian beam method. In relation to the asymptotic ray method, the Gaussian beam method is always finite at caustics. In addition, no prior knowledge of caustic locations is required as in the Maslov method [Kravtsov, 1968; Chapman and Drummond, 1982]. Since the Gaussian beam method relies on local para-

Copyright 1984 by the American Geophysical Union.

Paper number 4B0465.

0148-0227/84/004B-0465\$05.00 bolic approximations, no global direction of propagation must be assumed as in the standard parabolic approximation. Finally, the Gaussian beam method is comparable in cost to ray methods, and possibly even faster since no two-point ray tracing is done.

The organization of this paper is as follows. First, a more detailed description of the Gaussian beam method is given along with computational procedures. Then, various examples are given in order to illustrate different uses of the method as well as to test its validity. Two procedures are used to test the method. First, the reciprocal theorem, valid for an arbitrary heterogeneous medium, is applied. The discrepancy between reciprocal seismograms is considered as a measure of the error in the Gaussian beam solution. Second, the Gaussian beam synthesis is applied to several cases, including the twodimensional soft basin problem, for which solutions are known by other approximate methods. The Gaussian beam method is then used for two applications. First, the method is used to study volcanic earthquakes recorded under Mount Saint Helens. Then, the method is applied to scattering of teleseismic $\boldsymbol{P}$ waves by a lithosphere with randomly fluctuating velocities.

\section{Review of the Gaussian Beam Method}

In this section a more detailed description of the Gaussian beam method is given. The discussion is limited to the twodimensional case, for which the examples are given, and follows the results of Červeny et al. [1982], Červený and Pšenčik [1983b], and Červený [1983].

Before solving for the rays and the beam solutions, the velocity model must be parameterized. The velocities are input as discrete points and then interpolated using cubic splines. This results in continuous velocities as well as first and second derivatives. For velocities given on a mesh in two or three dimensions, this involves a tensor product of one-dimensional splines [De Boor, 1980]. Velocity discontinuities are included by introducing interfaces between layers with smoothly varying velocities. Curved interfaces are input as a discrete set of points and then spline interpolated. 


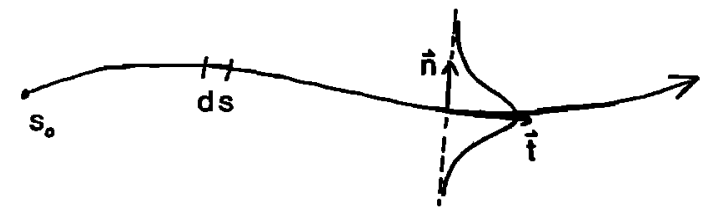

Fig. 1. Ray-centered coordinate system in two dimensions. The beam solution is concentrated near the ray and has a Gaussian shape.

Spline interpolation of the velocity may introduce some oscillation in the interpolated function depending on the input grid values [Azbel et al., 1980]. Care must be taken to check the interpolated function and to have the grid velocities change slowly. Several methods can be used to avoid the oscillations in the interpolating function. The first is to use smoothed splines as described by Pretlova [1976]. A second approach is to use splines under tension [see Cline, 1981]. For the remainder of this paper, careful use is made of simple bicubic spline interpolation by comparing the desired and interpolated functions.

The first step of the Gaussian beam method is to solve the kinematic ray tracing system. The rays are extremal of Fermat's integral and are determined by solving the ray equations:

$$
\frac{d x_{i}}{d s}=v p_{1} \quad \frac{d p_{i}}{d s}=-v^{-2} \frac{\partial v}{\partial x_{i}} \quad i=1,2
$$

with initial conditions

$$
\mathbf{x}\left(s_{0}\right)=\mathbf{x}_{0} \quad \mathbf{p}\left(s_{0}\right)=\mathbf{p}_{0}
$$

where $\mathbf{x}$ is the location along the ray and $\mathbf{p}$ is the slowness vector tangent to the ray. In an elastic medium, the velocity $v$ is either $\alpha$ or $\beta$. This system can be solved by standard numerical techniques such as the Runge-Kutta method. At an interface, Snell's law is applied locally. These ray equations are appropriate for isotropic nondispersive media. In isotropic dispersive media, wave packets will travel at the group velocity, but their trajectories will be determined by the local phase velocity.

The second step of the Gaussian beam method involves solving the wave equation locally in ray-centered coordinates using the parabolic approximation. The ray-centered coordinate system is an orthogonal curvilinear system that follows along a particular ray and was introduced to seismology by Popov and Pšenčik [1976, 1978]. In a two-dimensional medium, the ray-centered coordinates can be specified by the unit vector $t$ tangent to the ray and the unit vector $n$ normal to the ray (see Figure 1). An element of infinitesimal length in the ray-centered coordinates can be written

$$
|d r|^{2}=h_{1}{ }^{2} d s^{2}+h_{2}{ }^{2} d n^{2}
$$

where the scale factors $h_{1}$ and $h_{2}$ are given by

$$
h_{1}=1+v^{-1} v_{n} n \quad h_{2}=1
$$

where $v$ and $v_{n}$ are evaluated on the central ray with $n=0$.
The ray-centered coordinates comprise an orthogonal system. Since the local flow of high-frequency energy is along rays, the ray-centered coordinate system is an appropriate system for approximating the wave equation by local one-way operators.

The elastodynamic equation is then written in orthogonal curvilinear coordinates, as in $A k i$ and Richards [1980, equation 2.48], using scale factors for the ray-centered system. The basic step in deriving a one-way operator from the elastodynamic equation is the substitution

$$
u_{j}(s, n, \omega, t)=U_{j}(s, n, \omega) e^{-i \omega(t-t(s))}
$$

where $\tau(s)=\int v(s)^{-1} d s$ is the time delay along the central ray, $v(s)=\alpha$ or $v(s)=\beta$, and $U_{j}$ is a slowly varying envelope. The envelope is then expanded as

$$
U_{j}=\sum_{i=0}^{\infty} U_{j}^{i} \omega^{-i / 2}
$$

Note that this is in half powers of $\omega^{-1}$ as opposed to the typical ray series expansion in integral powers. In two dimensions, $U_{s}$ and $U_{n}$ are the in-plane components along the ray and normal to it, and $U_{b}$ is the out of plane component normal to the ray. After substitution of (3) into the elastodynamic equation, the resulting equations are approximated for large $\omega$. The following results are obtained by retaining only terms of order $\omega^{\gamma}, \gamma \geq 1$ and letting $\nu=\omega^{1 / 2} n$ assuming that $v=O(1)$. To this order of approximation, the $P$ and $S$ components decouple. For an incident $P$ wave [Cervený and $P \check{s} e n c ̌ i k$, 1983b],

$$
\begin{gathered}
U_{s}{ }^{0}(s, v)=\frac{1}{[\alpha(s) \rho(s)]^{1 / 2}} W^{\alpha}(s, v) \quad U_{n}^{0}=0 \\
U_{n}{ }^{1}=-i \alpha U_{s, \nu}{ }^{0} \quad U_{b}=0
\end{gathered}
$$

where $W^{a}$ satisfies the following parabolic equation:

$$
2 i \alpha^{-1} W_{, s}^{\alpha}+W_{, v \nu}{ }^{2}-\alpha^{-3} \alpha_{., n n} v^{2} W^{\alpha}=0
$$

For an incident $S V$ wave

$$
\begin{gathered}
U_{n}{ }^{0}(s, v)=\frac{1}{[\beta(s) \rho(s)]^{1 / 2}} W^{\beta}(s, v) \quad U_{s}{ }^{0}=0 \\
U_{s}{ }^{1}=i \beta U_{n, v}{ }^{0} \quad U_{b}=0
\end{gathered}
$$

where $W^{\beta}$ satisfies the parabolic equation

$$
2 i \beta^{-1} W_{, s}^{\beta}+W_{, v v}{ }^{\beta}-\beta^{-3} \beta_{, n n} v^{2} W^{\beta}=0
$$

and for an incident $S H$ wave

$$
U_{b}{ }^{0}(s, v)=\frac{1}{[\beta(s) \rho(s)]^{1 / 2}} W^{\beta}(s, v) \quad U_{s}=U_{n}=0
$$

with $W^{\beta}$ satisfying the same parabolic equation as for the $S V$ case. All velocities and densities are evaluated along the central ray. These results were derived from asymptotic analysis of the elastodynamic equation. The same basic parabolic equation for $W$ can also be obtained from the eikonal equation as shown in Appendix A.

The last term in the parabolic equation (5) or (7) comes from the approximation of order $\omega$ :

$$
h_{1} \omega^{2}\left[\frac{1}{v(s, n)^{2}}-\frac{1}{h_{1}^{2} v(s)^{2}}\right] W \approx-\omega v(s)^{-3} v_{, n n} v^{2} W
$$

where $v(s)$ and $v_{, n n}$ are evaluated along the central ray, $v(s, n)$ is the velocity in the vicinity of the central ray, and $h_{1}$ is the scale factor for the ray-centered coordinates. Without this ap- 
proximation for the last term, the parabolic equations (5) and (7) are similar in form to those given by $A k i$ and Richards [1980, equation 13.154] or Claerbout [1976, equation 10-3-9]. The parabolic equations (5) and (7) are valid for small-angle forward scattering about the central ray direction assuming large-scale inhomogeneities with respect to the wavelength. In addition, the validity of the above approximation requires that the velocities be smoothly varying within a certain beam width of the central ray. Since, as will be seen, the beam solutions to (5) or (7) spread with distance, this will result in a range limitation for the Gaussian beam method.

A particular solution to the parabolic equation (5) or (7) can be written as

$$
W(s)=\frac{1}{[q(s)]^{1 / 2}} \exp \left(\frac{i}{2} \frac{p(s)}{q(s)} v^{2}\right)
$$

where $q(s)$ and $p(s)$ satisfy

$$
\frac{d}{d s}\left[\begin{array}{l}
q \\
p
\end{array}\right]=\left[\begin{array}{cc}
0 & v \\
-v^{-2} v_{, n n} & 0
\end{array}\right]\left[\begin{array}{l}
q \\
p
\end{array}\right]
$$

with $v(s)$ and $u_{, n n}$ evaluated along the central ray. These are the same equations as the dynamic ray tracing system for ray theory, except here $p(s)$ and $q(s)$ must be complex. The variables $p(s)$ and $q(s)$ must also satisfy certain conditions, among them the condition $\operatorname{Im}(p / q)>0$. In this case the energy will be confined to a vicinity of the central ray, and the solution will be a Gaussian beam (see Figure 1). For ray theory, $p$ and $q$ are real and $q(s)$ has the interpretation of spreading and $v(s)$. $[p(s)] /[q(s)]$ has the interpretation of wave front curvature. The equations in (10) are in transmission-line form, where the ratio $p / q$ is related to the characteristic admittance for the system.

The complete solution to the parabolic equation for a particular central ray is made up of an infinite system of linearly independent beam solutions or modes [Červený et al., 1982] of the form

$$
\begin{aligned}
W_{k}(s, v)=\frac{1}{[q(s)]^{1 / 2}}\left(\frac{q^{*}}{q}\right)^{k / 2} H_{k}\left\{v[\operatorname{Im}(p / q)]^{1 / 2}\right\} \\
\cdot \exp \left(\frac{i}{2} \frac{p}{q} v^{2}\right) \quad k=0,1,2, \cdots
\end{aligned}
$$

where $H_{x}$ are hermite polynomials. Here only the zeroth order beam solution (9) is used for each central ray and no mode coupling between higher-order beam solutions is assumed.

The localized solution to the two-dimensional elastodynamic equation along a particular central ray, specified by a ray index parameter $\delta$, can be written in the following form [Červený and P̌̌enčik, 1983b]:

$$
\begin{aligned}
& \mathbf{u}_{\delta}(s, n, \omega)=\frac{1}{[v(s) \rho(s) q(s)]^{1 / 2}} \mathbf{r} \\
& \cdot \exp \left[-i \omega\left(t-\int_{0}^{s} \frac{d s}{v(s)}-\frac{1}{2} \frac{p(s)}{q(s)} n^{2}\right)\right]
\end{aligned}
$$

where for $P$ waves,

$$
v(s)=\alpha(s) \quad \mathbf{r}=\left[\mathbf{t}+\mathbf{n} \alpha(s) \frac{p(s)}{q(s)} n\right]
$$

for $S V$ waves,

$$
v(s)=\beta(s) \quad \mathbf{r}=\left[\mathbf{n}-\mathbf{t} \beta(s) \frac{p(s)}{q(s)} n\right]
$$

and for $S H$ waves, $v(s)=\beta(s)$ and $\mathbf{r}=\mathbf{b}$ (out of plane component), where $p$ and $q$ solve (10) and are, in general, complex, and $\mathbf{r}$ gives the orientation of the displacement vector near the central ray. Note that the beam solutions are valid only where the ray-centered coordinates are well defined and regular in some region near the central ray.

The beam solutions must be modified in the presence of any interfaces. Following Popov [1982], it is assumed that to first approximation the beam solutions (11) for a given ray remain uncoupled at a smoothly curved interface. Then the particular beam solution (9) is only modified by a possibly complex reflection or transmission coefficient and the change in $p(s)$ and $q(s)$ due to the interface. Formulas for how real-valued $p(s)$ and $q(s)$ transform at an interface are given by $\check{C}_{\text {erveny }}$ and Pšenčik [1979], and for curvature by Červený and Hron [1980]. Formulas for how complex-valued $p(s)$ and $q(s)$ transform at an interface are given by V. Cervený and I. Pšenčik (unpublished manuscript, 1984), where the equations are written so that $q(s)$ is a relative spreading across each interface. This avoids writing an additional factor in the beam amplitude.

For Gaussian beams, $p(s)$ and $q(s)$ can be written as [see Cervený et al., 1982]

$$
q(s)=\varepsilon q_{1}(s)+q_{2}(s) \quad p(s)=\varepsilon p_{1}(s)+p_{2}(s)
$$

where $p(s)$ and $q(s)$ satisfy (10), $\varepsilon$ is a complex valued parameter to be determined, and

$$
\left[\begin{array}{ll}
q_{1} & q_{2} \\
p_{1} & p_{2}
\end{array}\right]
$$

is the fundamental matrix of linearly independent real solutions of (10) with initial conditions

$$
\left[\begin{array}{cc}
1 & 0 \\
0 & 1 / v_{0}
\end{array}\right]
$$

From these initial conditions,

$$
K_{1}\left(s_{0}\right)=v_{0} \frac{p_{1}\left(s_{0}\right)}{q_{1}\left(s_{0}\right)}=0
$$

representing an initial plane wave at the source,

$$
K_{2}\left(s_{0}\right)=v_{0} \frac{p_{2}\left(s_{0}\right)}{q_{2}\left(s_{0}\right)}=\infty
$$

representing a line source, where $v_{0}=v\left(s_{0}\right)$. Thus the complex solution is a linear combination of real solutions for a plane wave and line source weighted by $\varepsilon$ as in (13). Here $\varepsilon$ is a factor chosen so that $(a) q(s) \neq 0$, resulting in no singularities in amplitude along the ray, and $(b)$

$$
\operatorname{Im}\left(\frac{p(s)}{q(s)}\right)>0
$$

giving a solution that is concentrated near the ray. Following Cerveny et al. [1982], $\varepsilon$ is written in the form

$$
\varepsilon=S_{0}-i \frac{\omega}{2 v_{0}} L_{M}^{2}
$$

where, geometrically, $L_{M}$ is the beam width at a specific location along the beam known as the beam waist and $S_{0}$ is a parameter that shifts the location of the beam waist away from the source location, $s_{0}$, along the ray. Cerveny et al. [1982] showed that conditions (a) and (b) above are both satisfied when $L_{M} \neq 0$. This result depends on the fact that the 
determinant of the fundamental matrix above is a nonzero constant along the ray and therefore $q_{1}(s)$ and $q_{2}(s)$ cannot simultaneously be zero for a given $s$. Thus the method is constructed to ensure no singularities in amplitude.

The fundamental beam solution can now be written from (12) as

$$
\begin{aligned}
& \mathbf{u}_{\delta}=\frac{1}{[v(s) \rho(s) q(s)]^{1 / 2}} \mathbf{r} \\
& \quad \cdot \exp \left[-i \omega\left(t-\int_{0}^{s} \frac{d s}{v(s)}\right)+\frac{i \omega}{2 v} K(s) n^{2}-\frac{n^{2}}{L(s)}\right]
\end{aligned}
$$

where

$$
q(s)=\left[S_{0}-i \frac{\omega}{2 v_{0}} L_{M}^{2}\right] q_{1}+q_{2}
$$

equal to the complex spreading,

$$
K(s)=v(s) \operatorname{Re}\left[\frac{p(s)}{q(s)}\right]
$$

equal to the phase front curvature of the beam, which reduces to

$$
K(s)=\frac{v(s)\left[\left(S_{0} p_{1}+p_{2}\right)\left(S_{0} q_{1}+q_{2}\right)+\left(\frac{\omega L_{M}{ }^{2}}{2 v_{0}}\right)^{2} p_{1} q_{1}\right]}{\left[\left(S_{0} q_{1}+q_{2}\right)^{2}+\left(\frac{\omega L_{M}{ }^{2}}{2 v_{0}}\right)^{2} q_{1}^{2}\right]}
$$

and

$$
L(s)=\left[\frac{\omega}{2} \operatorname{Im}\left(\frac{p(s)}{q(s)}\right)\right]^{-1 / 2}
$$

equal to the beam halfwidth, which reduces to

$$
L(s)=\left[L_{M}{ }^{2} q_{1}^{2}+\left(\frac{2 v_{0}}{\omega L_{M}}\right)^{2}\left(S_{0} q_{1}+q_{2}\right)^{2}\right]^{1 / 2}
$$

For convenience, the parameter $L_{0}$ will sometimes be used to specify the initial beam width at the beam waist. $L_{0}$ is specified by $L_{M}=\left(2 v_{0} / \omega\right)^{1 / 2} L_{0}$, and is independent of frequency since from (18), $L_{M}=L\left(s_{0}-S_{0}\right)=O\left(\omega^{-1 / 2}\right)$.

The properties of a Gaussian beam in a homogeneous medium are briefly reviewed in Appendix B. An example of a Gaussian beam in a homogeneous medium with $L_{M}=1$ and $\lambda=\pi / 4$ is shown in Figure 2. The beam waist is located at $s=0$. The solid lines show the spread of the beam with distance, and the dashed straight lines are the far-field asymptotes. The distance $s_{c}$ separates the collimated near zone from the diverging far zone of the beam and is proportional to the initial beam width squared over the wavelength. The near zone has planar phase fronts, while the far zone diverges as if from a point. By adjusting the initial beam width, the beam width observed at a given station range can be changed. Adjusting the initial beam width so that the station distance is just $s_{c}$ results in the smallest possible beam width at the station. This will be called the critical initial beam width for a given station range. It also places the station just between the collimated planar part of the beam and the far-field spreading part. For a homogeneous medium the critical initial beam width is $L_{M_{c r}}=(\lambda s / \pi)^{1 / 2}$. For the general inhomogeneous case,

$$
L_{M_{\mathrm{cr}}}=\left(\frac{2 v_{0}}{\omega}\right)^{1 / 2}\left(\left|\frac{S_{0} q_{1}+q_{2}}{q_{1}}\right|\right)^{1 / 2}
$$

[see Červený et al., 1982].

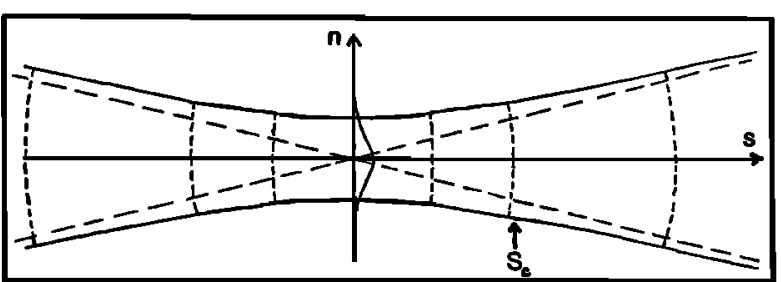

Fig. 2. A Gaussian beam in a homogeneous medium with $L_{M}=1$ and $\lambda=\pi / 4$.

An alternative way to generate a Gaussian beam in a homogeneous medium is to use an initial planar Gaussian amplitude distribution and diffract it using the Fresnel diffraction integral [see Marcuse, 1982]. Since the Gaussian beam is a known solution to the Fresnel integral for a given initial amplitude distribution, the field from an arbitrary initial amplitude distribution can be approximated as a sum of basis Gaussian beams.

The final step of the Gaussian beam method is a superposition of beam solutions to represent a given source using the proper weight functions $\Phi(\delta)$, where $\delta$ is the ray index parameter. For an initial line source, $\delta$ represents an initial takeoff angle for a given ray. For an initial plane wave, $\delta$ represents the distance along the wave front for a given ray. Each beam solution solves the wave equation in the vicinity of a ray, and together, the weighted superposition approximately satisfies a source condition for a given source. Thus the following integral over ray index parameter and frequency can be written

$$
\mathbf{u}(\mathbf{x}, t)=\frac{1}{\pi} \operatorname{Re} \int_{0}^{\infty} \int_{\delta_{0}}^{\delta_{M}} \Phi(\delta) F(\omega) \mathbf{u}_{\delta}(s, n, \omega, t) d \delta d \omega
$$

where $F(\omega)$ is the source spectra and $\delta_{0}, \delta_{M}$ define the range of the ray index parameter. The values $\delta_{0}$ and $\delta_{M}$ must be chosen to adequately cover the area of interest with rays. Using the slowness approach [Chapman, 1978],

$$
\mathbf{u}(\mathbf{x}, t)=\int_{\delta_{0}}^{\delta_{M}} \Phi(\delta) g(x, t, \delta) d \delta
$$

where $\mathbf{g}(\mathbf{x}, t, \delta)$ is a wave packet [see Cervený, 1983]. In the examples, $F(\omega)$ is the source spectra for the following damped cosine wavelet [see Cervený et al., 1977]:

$$
f(t)=\exp \left[-(\omega t / \gamma)^{2}\right] \cos \left(\omega t+\varphi_{0}\right)
$$

Using this source wavelet, the wave packet $g(x, t, \delta)$ is approximately Gaussian in both time and space [Červený, 1983]. Equation (21) is then approximated by the finite sum

$$
\mathbf{u}(\mathbf{x}, t)=\sum_{t=0}^{N} \Phi\left(\delta_{i}\right) \mathrm{g}\left(\mathbf{x}, t, \delta_{i}\right) \Delta \delta
$$

where $\Delta \delta$ must be chosen small enough to smoothly interpolate the beam solutions and to adequately sample the medium.

The weight factors $\Phi(\delta)$ for a given source must then be specified. The weight function for an acoustic line source is given by Popov [1982] and Červený et al. [1982], and for a plane wave by Červený [1982]. In Appendix $C$ the weight function for a two-dimensional point force in an elastic medium is obtained by comparing the Gaussian beam superposition with the exact plane wave decomposition.

There are thus several free parameters when computing the Gaussian beam solution for a given problem: $\delta_{0}, \delta_{M}$, and $\Delta \delta$ for the finite sum, the initial beam width at the beam waist 
$L_{M}$, and the shift of the beam waist away from the source given by the parameter $S_{0}$. The sensitivity of the method on these parameters, particularly $L_{M}$, will be investigated in several of the following examples.

\section{Applications}

In this section, several applications of the Gaussian beam method are given in order to illustrate the used of the method and test its range of applicability. The computer program for the Gaussian beam synthesis was modified from the ray tracing program, RAY81, written by Pšenčik [1983]. For all examples, the slowness method is used in which the frequency integral is evaluated first assuming Gaussian packets and a damped cosine source wavelet [Červený, 1983].

\section{Example $A$}

In this example, an initial plane wave in a homogeneous medium is decomposed into a number of centered Gaussian beams. Each beam is propagated along the straight line rays shown in Figure $3 a$. The initial plane wave is located at $z=$ $-50 \mathrm{~km}$. The station is shown by the triangle at $x=25 \mathrm{~km}$, $z=0 \mathrm{~km}$. The velocity is $6 \mathrm{~km} / \mathrm{s}$, and the source wavelet parameters are $f=2 \mathrm{~Hz}, \gamma=4, \varphi_{0}=0$ (see (22)). The contribution of each beam to the resulting sum is shown for three different initial beam widths in Figures $3 b-3 d$. The initial beam width is represented by $L_{0}$, a frequency independent parameter where $L_{M}=\left(2 v_{0} / \omega\right)^{1 / 2} L_{0} . L_{M}$ is in kilometers, and $L_{0}$ is in $\mathrm{km}^{1 / 2}$. For this example, $L_{M}=0.98 L_{0}$. The value $L_{0}=7 \mathrm{~km}^{1 / 2}$ represents the critical initial beam width that gives the smallest beam width at the station range of $50 \mathrm{~km}$. Larger than critical initial beam widths result in planar beams
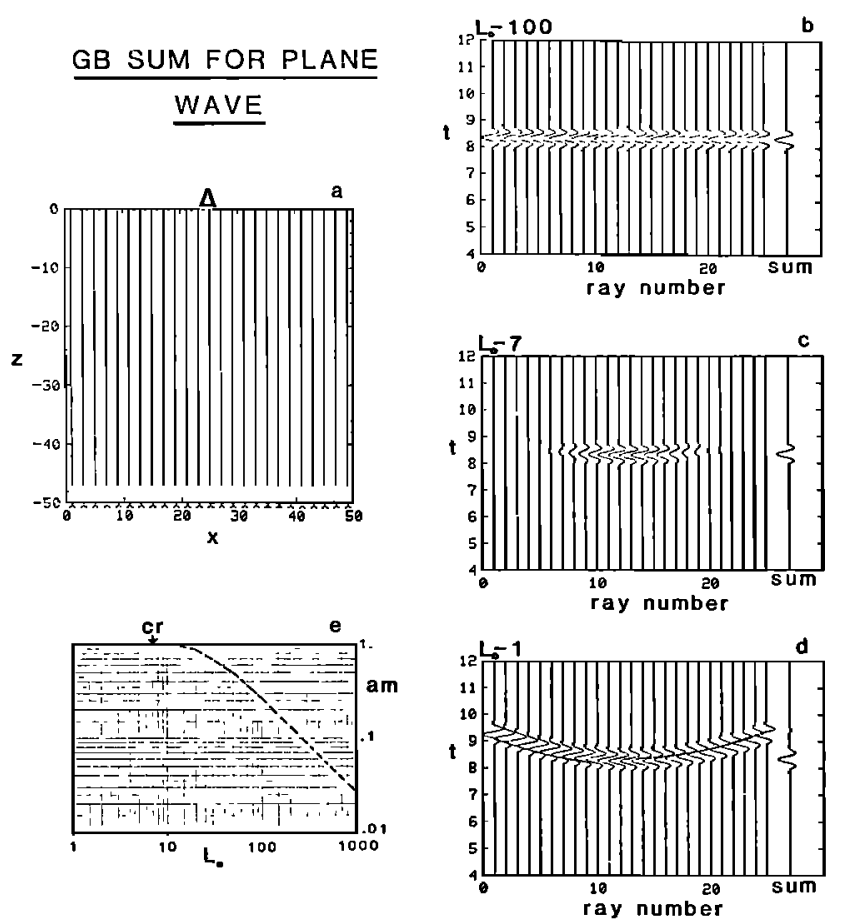

Fig. 3. Superposition of Gaussian beams to represent an initial plane wave in a homogeneous medium. (a) Ray diagram for initial plane wave at $z=50 \mathrm{~km}$. Individual beam contributions indexed by ray number along the wave front and the resulting sum for three initial beam widths. (b) $L_{0}=100 \mathrm{~km}^{1 / 2},(c) L_{0}=7 \mathrm{~km}^{1 / 2}$, and $(d)$ $L_{0}=1 \mathrm{~km}^{1 / 2}$. (e) The amplitude of the Gaussian beam sum as a function of $L_{0}$. at the receiver, while smaller then critical initial beam widths result in diverging phase fronts at the receiver as if from a point.

The "ray number" in Figures $3 b-3 d$ represents the ray index parameter, which for this example measures distance along the initial plane wave front. The initial ray spacing is $2 \mathrm{~km}$. The rays in the ray diagram (Figure $3 a$ ) are assigned a number from 1 to 25 . The beam going directly to the station follows along ray number 13 . The scaled sum for each initial beam width is shown to the right in Figures $3 b-3 d$.

For $L_{0}=1 \mathrm{~km}^{1 / 2}$, less than critical, only a limited number of beams around the direct ray contribute to the resulting sum due to phase interference (see Figure $3 d$ ). There is also a spurious end effect in the resulting sum caused by lack of cancellation of the end beams. As the initial beam width is changed to $L_{0}=7 \mathrm{~km}^{1 / 2}$, near critical, again only a limited number of beams contribute to the resulting sum (see Figure $3 c$ ). This is now caused by amplitude decay of beams away from the station. The spurious end effects have been avoided in the resulting sum by using the critical initial beam width. Finally, for $L_{0}=100 \mathrm{~km}^{1 / 2}$, larger than critical, the beams are more planar at the station distance and no amplitude or phase interference limits the number of rays contributing to the resulting sum (Figure $3 b$ ). The sum will then depend on the ray aperture used.

Figure $3 e$ shows the amplitude of the resulting sum as a function of initial beam width. The critical initial beam width is shown. The true amplitude at the receiver is one. For larger than critical initial beam widths, the amplitude of the Gaussian beam sum falls off, since a finite aperture of rays is used.

The following comments can be made concerning decomposing an initial plane wave in a homogeneous medium into Gaussian beams. For larger than critical initial beam widths, the amplitude is not stable using a finite ray aperture. For near-critical initial beam widths, the result is stable by amplitude decay of beams away from the receiver. The end effects are avoided here by amplitude decay. For smaller than critical initial beam widths, a stable result occurs by phase interference, but spurious end effects are noticeable in the resulting sum. This is similar to a decomposition of the initial wavefront into point-like sources as viewed at the receiver.

\section{Example B}

In this example, an initial half plane is decomposed into Gaussian beams. The half plane is located at a depth of $2.5 \mathrm{~km}$ and terminates at $x=15 \mathrm{~km}$ as seen in Figure $4 a$. Receivers are located on the surface from 12 to $18 \mathrm{~km}$ with a spacing of $0.2 \mathrm{~km}$. The velocity is $2.5 \mathrm{~km} / \mathrm{s}$ and the source wavelet parameters are $f=20 \mathrm{~Hz}, \gamma=5, \varphi_{0}=\pi / 2$.

The initial beam width $L_{M}$ is related to $L_{0}$ by $L_{M}=0.2 L_{0}$. The critical value of $L_{0}$ for a receiver range of $2.5 \mathrm{~km}$ is $L_{0_{\mathrm{cr}}}=s^{1 / 2}=1.58 \mathrm{~km}^{1 / 2}$. Figure $4 b$ shows the resulting seismograms for $L_{0}=1.5 \mathrm{~km}^{1 / 2}$. Since the initial amplitude on the edge of the half plane goes to zero gradually over $0.3 \mathrm{~km}$, the amplitude at the receivers smoothly goes to zero at $x=15 \mathrm{~km}$ with no evident edge diffraction. When $L_{0}$ is reduced to 0.05 $\mathrm{km}^{1 / 2}$, the initial amplitude on the edge of the half plane goes to zero much more sharply. A diffracted wave from the edge of the half plane can now be seen (Figure 4c).

\section{Example $C$}

In this example, the decomposition of a line source in a homogeneous medium into Gaussian beams is investigated. 

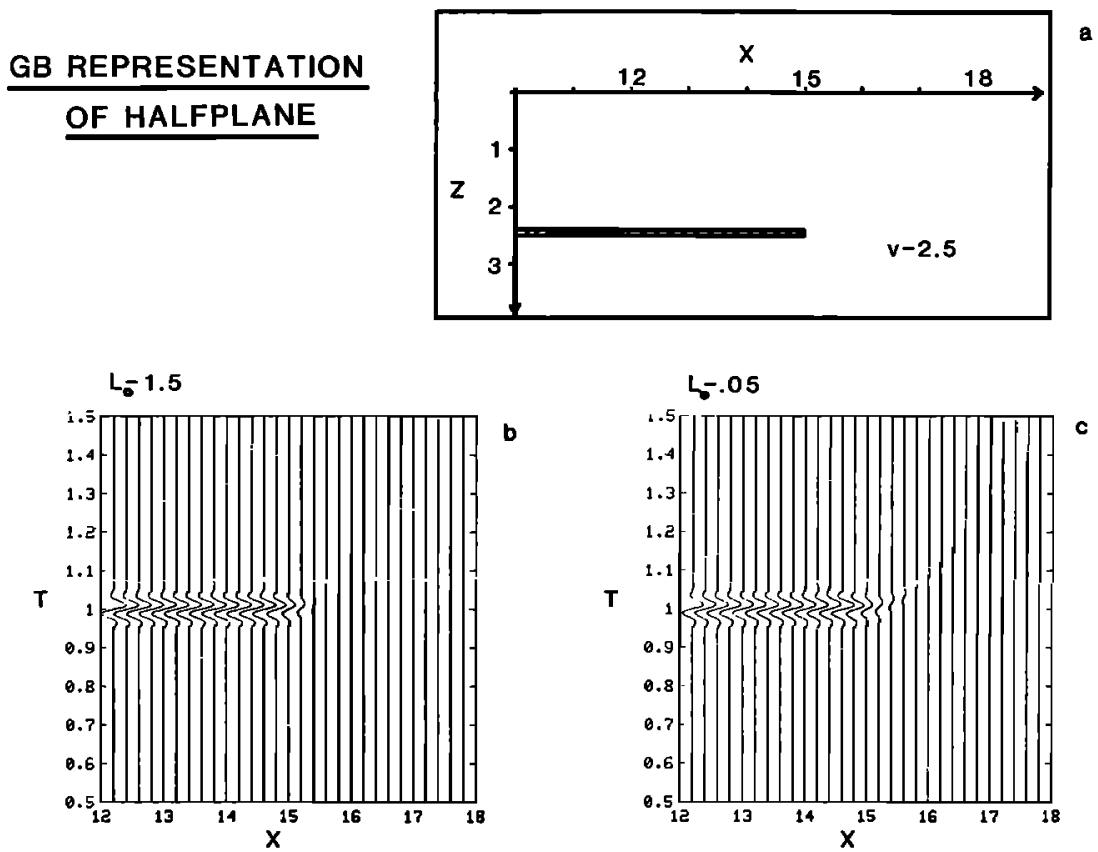

Fig. 4. Superposition of Gaussian beams to represent an initial half plane. (a) Half plane located at $z=2.5 \mathrm{~km}$ and terminating at $x=15 \mathrm{~km}$. Stations located on the surface from $x=12 \mathrm{~km}$ to $x=18 \mathrm{~km}$. (b) Gaussian beam seismograms for $L_{0}=1.5 \mathrm{~km}^{1 / 2}$. (c) Gaussian beam seismograms for $L_{0}=0.05 \mathrm{~km}^{1 / 2}$.

Each beam is propagated along the rays shown in the ray diagram in Figure $5 a$, for a source at $x=25 \mathrm{~km}, z=-50 \mathrm{~km}$. The station at which the resulting sum is evaluated is shown by the triangle located at $x=25 \mathrm{~km}, z=0 \mathrm{~km}$. The velocity is $6 \mathrm{~km} / \mathrm{s}$ and the source wavelet parameters are $f=2 \mathrm{~Hz}, \gamma=4$,
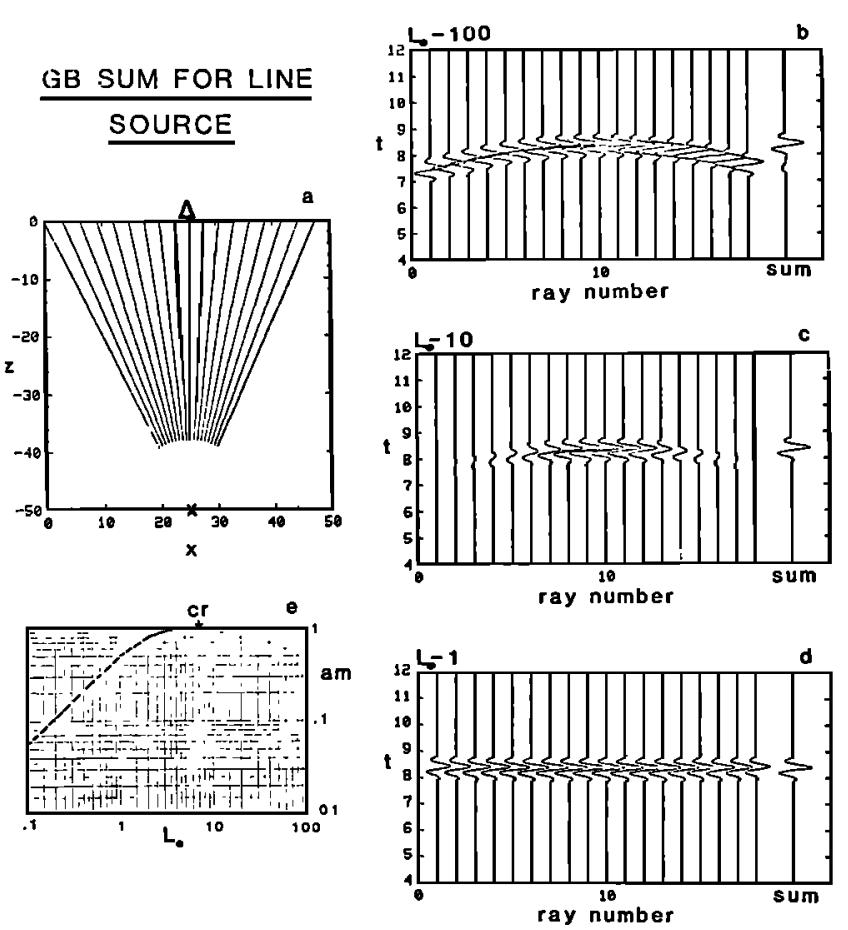

Fig. 5. Superposition of Gaussian beams to represent a line source in a homogeneous medium. (a) Ray diagram for line source at $x=25 \mathrm{~km}, z=-50 \mathrm{~km}$. Individual beam contributions indexed by ray number representing initial takeoff angle and the resulting sum for (b) $L_{0}=100 \mathrm{~km}^{1 / 2}$. (c) $L_{0}=10 \mathrm{~km}^{1 / 2}$, and $(d) L_{0}=1 \mathrm{~km}^{1 / 2}$. (e) The amplitude of the Gaussian beam sum as a function of $L_{0}$. $\varphi_{0}=0$. The contribution of each beam to the resulting sum at the station is shown for three initial beam widths in Figures $5 b-5 d$. The initial beam width is represented by $L_{0}$, where $L_{M}=0.98 L_{0}$. The ray number corresponds to the ray index parameter, which for this case is the initial takeoff angle at the source. The rays in the ray diagram (Figure $4 a$ ) are assigned a number from 1 to 18 . The beam going directly to the station is represented by ray number 10 . The scaled sum for each initial beam width is shown to the right in Figures $5 b-5 d$.

The initial beam width of $L_{0}=10 \mathrm{~km}^{1 / 2}$ is slightly larger than the critical value of $L_{0}=7 \mathrm{~km}^{1 / 2}$. For a given station distance, $L_{0}$ equal to critical separates planar beams from the beams that diverge as if from a point. For $L_{0}=100 \mathrm{~km}^{1 / 2}$, larger than critical, only a limited number of beams contribute around the direct ray due to phase interference (see Figure $5 b$ ). A noticeable end effect from the finite ray aperture can be seen in the sum. For $L_{0}=10 \mathrm{~km}^{1 / 2}$, again only a limited number of rays about the direct ray contribute to the sum (see Figure $5 c$ ). This is now due to amplitude decay of beams away from the station. The end effects have been avoided in this case. For $L_{0}=1 \mathrm{~km}^{1 / 2}$, smaller than critical, neither amplitude nor phase interference limits the number of beams contributing about the direct ray (see Figure $5 d$ ). The result then will depend on the ray aperture used.

The amplitude of the Gaussian beam seismogram as a function of initial beam width is shown in Figure $5 e$. The amplitude of 1.0 in Figure $5 e$ corresponds to the amplitude of the far-field approximation for a line source, where $U(D) \approx$ $-1 / 4 \pi(\lambda / r)^{1 / 2} e^{i \omega r / 0} e^{i \pi / 4}$. For $r=50 \mathrm{~km},|U(D)|=0.01949$. The amplitude of the Gaussian beam sum for $L_{0}=10 \mathrm{~km}^{1 / 2}$ is $\left|U_{G B}\right|=0.01958$. For larger than critical initial beam widths, the amplitude of the Gaussian beam synthesis is stable within $1 \%$ of the far-field line source solution. For smaller than critical initial beam widths, the amplitude of the Gaussian beam sum falls off.

The following conclusions can be made concerning decom- 
posing a line source into Gaussian beams. For larger than critical $L_{0}$, a stable result occurs by phase interference. This is similar to a decomposition of a line source into plane waves. Spurious end effects occur for this case due to the finite ray aperture. Using the critical $L_{0}$, a stable result occurs by amplitude decay and end affects are avoided. Finally, for smaller than critical $L_{0}$, the amplitude is not stable.

\section{Example $D$}

In this example, an $S H$ line source above a welded interface is investigated. The velocities are $3.7 \mathrm{~km} / \mathrm{s}$ over $4.6 \mathrm{~km} / \mathrm{s}$ with the source and receivers located $30 \mathrm{~km}$ above the interface. The receivers range from 20 to $200 \mathrm{~km}$ with a spacing of 20 $\mathrm{km}$. The model is shown in Figure $6 a$.

Figure $6 b$ shows the seismograms computed using the Cagniard method [see Aki and Richards, 1980]. The Cagniard result is convolved with a source wavelet with parameters, $f=3 \mathrm{~Hz}, \gamma=4, \varphi_{0}=0$, and the seismograms are plotted in reduced time, $T-X / 4.6$. The precritical reflection coefficient goes to zero near $60 \mathrm{~km}$ and then changes sign. The critical distance is located at $81.2 \mathrm{~km}$, and the amplitude becomes large at a distance slightly greater than the critical distance. For postcritical distances, total reflection occurs with a phase shift. A small head wave can also be seen emerging past the critical distance. The Gaussian beam result using a larger than critical initial beam width, $L_{0}=75 \mathrm{~km}^{1 / 2}$, is shown in Figure $6 c$. The Gaussian beam result is similar to the Cagniard result, including the head wave phase. But there also exists a spurious end effect cutting across the Gaussian beam seismograms from about $6 \mathrm{~s}$ at $100 \mathrm{~km}$ to about $12 \mathrm{~s}$ at $200 \mathrm{~km}$.
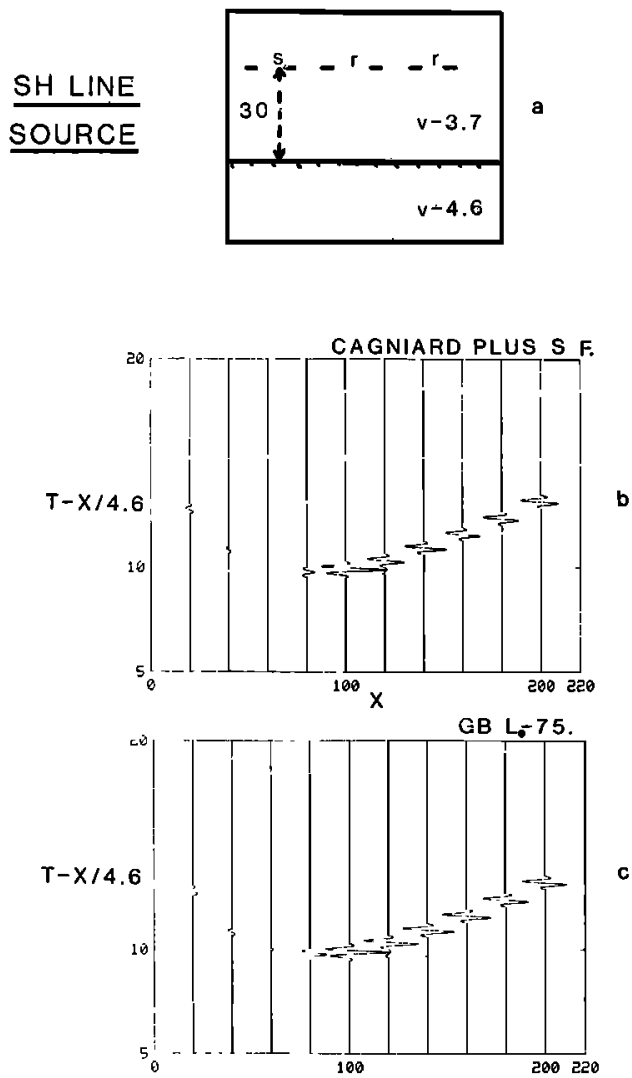

Fig. 6. SH line source above a welded interface. (a) Model geometry with source and receivers $30 \mathrm{~km}$ above interface. (b) Cagniard seismograms. (c) Gaussian beam seismograms using $L_{0}=75 \mathrm{~km}^{1 / 2}$.
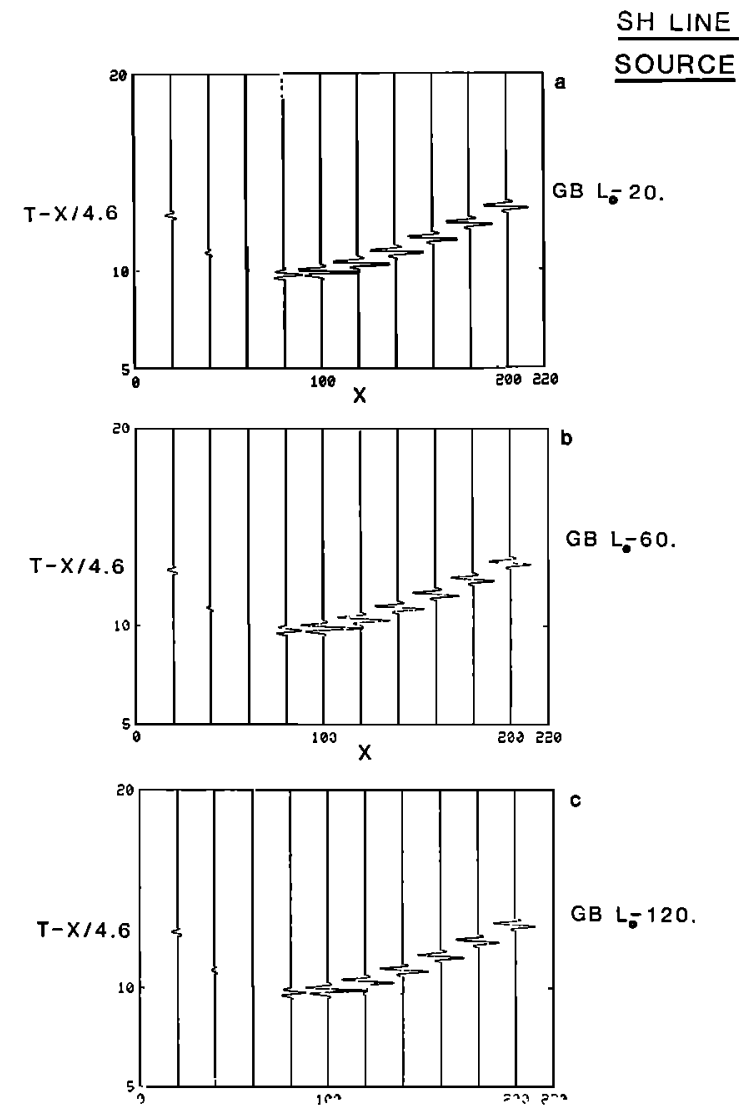

Fig. 7. Gaussian beam seismograms for $S H$ line source above welded interface for (a) $L_{0}=20 \mathrm{~km}^{1 / 2}$, (b) $L_{0}=60 \mathrm{~km}^{1 / 2}$, and (c) $L_{0}=120 \mathrm{~km}^{1 / 2}$.

The Gaussian beam result for three initial beam widths is shown in Figures $7 a-7 c$. The value of $L_{0}$ equal to 20 is slightly larger than critical for the farthest station. For $L_{0}=20 \mathrm{~km}^{1 / 2}$, the small head wave phase is not generated, but the other phases compare with the Cagniard result. For larger $L_{0}$, the head wave begins to form. In addition, an unwanted end effect begins to appear.

\section{Example E}

In this example, the effect of a single caustic in a layer over a gradient is reviewed. This is a standard example but illustrates several features of the Gaussian beam solution [see Červený et al., 1977, 1982; L. Klimeš and V. Cervený, unpublished manuscript, 1983]. The velocity model is constant to 15 $\mathrm{km}$ with a velocity of $5.6 \mathrm{~km} / \mathrm{s}$ and increases linearly from 15 $\mathrm{km}$ down to $40 \mathrm{~km}$ with a velocity of $8 \mathrm{~km} / \mathrm{s}$. The ray diagram is shown in Figure $8 a$ using 36 rays and a ray spacing of $1^{\circ}$. The wavelet parameters are $f=4 \mathrm{~Hz}, \gamma=3, \varphi_{0}=0.0$.

A caustic intersects the surface at $120 \mathrm{~km}$. Ray theory would predict an infinite amplitude at the caustic and no energy in the caustic shadow. The Gaussian beam seismograms are shown in Figures $8 b-8 c$ for two initial beam widths, $L_{0}=8 \mathrm{~km}^{1 / 2}$, near the average critical value for the set of rays, and $L_{0}=128 \mathrm{~km}^{1 / 2} . S_{0}$ is chosen to be 0.0 , locating the beam waist at the source. The Gaussian beam seismograms show several interesting features including the shift of the peak amplitude to the right of the caustic location and the penetration of energy into the caustic shadow. As a result of phase interference between beams, the $L_{0}=128 \mathrm{~km}^{1 / 2}$ does not pen- 


\section{LAYER OVER GRADIENT}
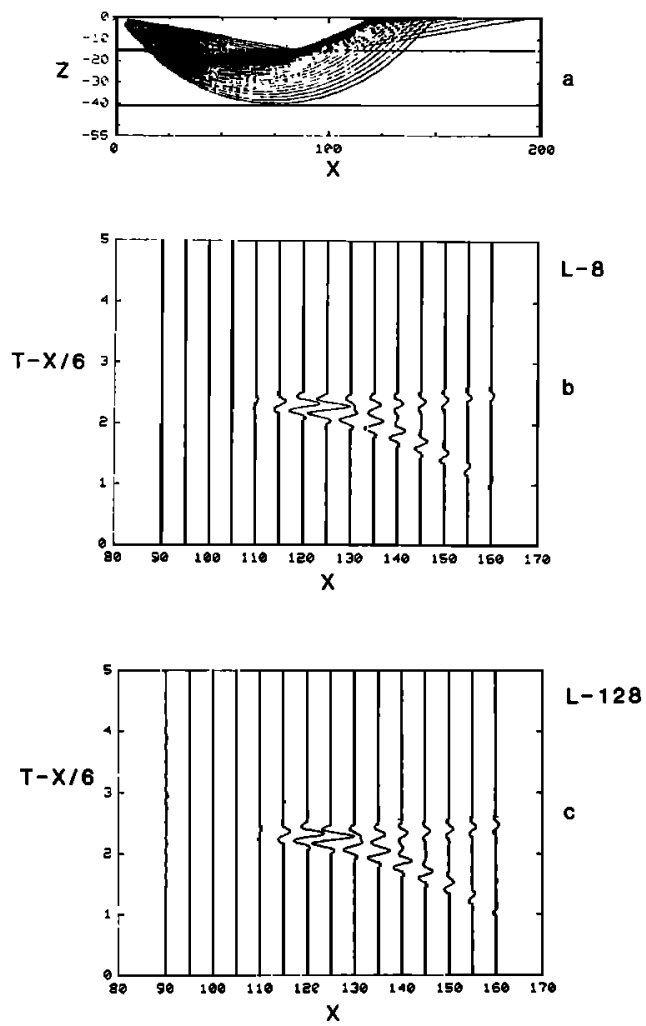

Fig. 8. Layer over a gradient. (a) Ray diagram where caustic intersects surface at $x=120 \mathrm{~km}$. (b) Gaussian beam seismograms for $L_{0}=8 \mathrm{~km}^{1 / 2}$. (c) Gaussian beam seismograms for $L_{0}=128 \mathrm{~km}^{1 / 2}$.

etrate any further into the caustic shadow than the $L_{0}=8$ $\mathrm{km}^{1 / 2}$ solution. But spurious phases can be seen in the $L_{0}=$ $128 \mathrm{~km}^{1 / 2}$ solution that do not quite cancel with the ray set used.

In order to investigate how each beam contributes to the final seismograms the individual beam solutions are plotted for several station locations and initial beam widths. The results for a station at $140 \mathrm{~km}$ are shown in Figure 9. Figure $9 a$ shows the ray diagram using 18 rays and a ray spacing of $2^{\circ}$. The station is located by the triangle, and two geometric arrivals are seen at this station. Figures $9 b-9 c$ show the individual beam contributions for $L_{0}=8$ and $128 \mathrm{~km}^{1 / 2}$. The ray number represents the ray index parameter. The rays are here simply indexed from 1 to 18 from a shallow initial takeoff angle to a steep initial takeoff angle from horizontal. The two geometric arrivals are seen in the scaled sum to the right in Figure $9 b-9 c$.

For $L_{0}=8 \mathrm{~km}^{1 / 2}$, near the average critical value, the energy is concentrated around the two direct beams to the station with ray numbers 4 and 17. The individual beam wavelets in Figure $9 b$ map out a curve that has stationary points corresponding to the direct beams to the station. For $L_{0}=128 \mathrm{~km}^{1 / 2}$, the energy is more evenly distributed among the beams as seen in Figure $9 c$. The beam solutions away from the stationary points now phase interfere, giving a similar resulting sum as for the $L_{0}=8 \mathrm{~km}^{1 / 2}$ case.

For very large $L_{0}$, each beam solution is effectively an initial plane wave contribution. For a large $L_{0}$ and a onedimensional medium, the individual beam contributions in phase time and ray number are similar to plạne wave decompositions in phase time, $\Theta=\tau+p x$, and horizontal ray parameter [see Chapman, 1978].

Figure 10 shows the results for a station at $x=120 \mathrm{~km}$, where the caustic intersects the surface. Here the two geometric arrivals merge as one. Figure $10 b$ shows the individual beam contributions for $L_{0}=8 \mathrm{~km}^{1 / 2}$. Again the ray number represents the ray index parameter and indexes the rays from 1 to 18 . There is now only one stationary point in the curve, represented by the locus of individual beam wavelets, located at ray number 10 . The result for $L_{0}=128 \mathrm{~km}^{1 / 2}$ is shown in Figure $10 c$. The sum shows a spurious end effect caused by the lack of cancellation of the end beams. The $L_{0}=8 \mathrm{~km}^{1 / 2}$ case avoids this end effect by amplitude decay of beams away from the station.

In Figure 11, the beam contributions are shown for a station at $x=110 \mathrm{~km}$ in the caustic shadow. Here no geometric arrival occurs, and the individual beam wavelets shown in Figures $11 b-11 c$ have no stationary points. The $L_{0}=8 \mathrm{~km}^{1 / 2}$ case shows a clean resulting sum, whereas the $L_{0}=128 \mathrm{~km}^{1 / 2}$ case shows spurious end effects that are the same order as the actual signal.

\section{Example F}

In this example the effect of caustics on $q(s)$ is investigated. The caustics result from multiple bounces of rays in a surface wave guide. The velocity model consists of a $0.1-\mathrm{km}$ homogeneous surface layer having a velocity of $1.8 \mathrm{~km} / \mathrm{s}$ over a linear velocity gradient from 1.8 to $5.6 \mathrm{~km} / \mathrm{s}$ at $5.0 \mathrm{~km}$. The ray diagram for a source in the thin surface layer is shown in

\section{LAYER OVER GRADIENT}
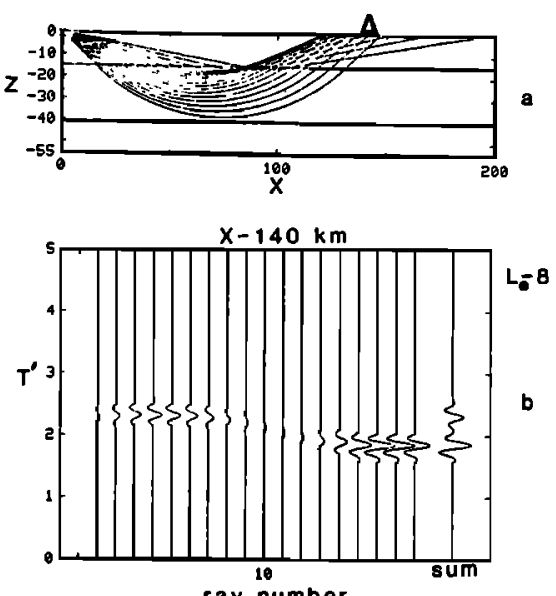

ray number

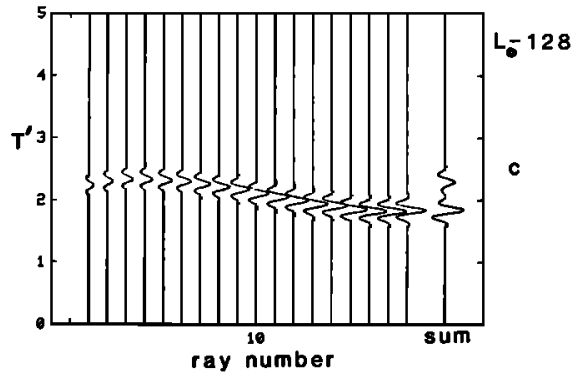

Fig. 9. Layer over a gradient. (a) Ray diagram with station at $x=140 \mathrm{~km}$. (b) Individual beam contributions and resulting sum for $L_{0}=8 \mathrm{~km}^{1 / 2}$. (c) Individual beam contributions and resulting sum for $L_{0}=128 \mathrm{~km}^{\mathrm{i} / 2}$. 
Figure $12 a$. The complex function $q^{\mathrm{r}}(s)$ is shown in Figure $12 b$ as a function of distance along the ray. Here $q^{r}(s)$ is a relative spreading across each interface, and for this case is related to $q(s)$ by

$$
q^{\mathrm{r}}(s)=(-1)^{\alpha_{\mathrm{s}}} q(s)=(-1)^{\alpha_{\mathrm{r}}}\left[\varepsilon q_{1}(s)+q_{2}(s)\right]
$$

where $\alpha_{r}$ is the number of reflections from an interface. Here $q^{r}(s)$ is the actual spreading factor that occurs in the amplitude of the beam [see Červený and Pšenčik, 1983a]. For simplicity, the parameter $\varepsilon$ is set to $-i$. The horizontal scale for $q^{\mathrm{r}}(s)$ is 10 times larger than the vertical scale.

For ray theory, caustics occur when $q_{2}(s)=\operatorname{Re}[q(s)]$ goes to zero. Several zero crossings for $\operatorname{Re}\left[q^{r}(s)\right]$ are shown in Figure $12 b$, for example, between points $b$ and $c$. By combining the results for an initial plane wave and an initial line source, the magnitude of the complex function $q^{r}(s)$ never equals zero. In Figure $12 b$ the plot of $q^{r}(s)$ cycles counterclockwise about the origin but never passes through it.

Using ray theory, a phase advance of $\pi / 2$ must be introduced at each caustic. This is done using the KMAH index, which counts the number of caustics crossed by a given ray. Using a complex $q^{r}(s)$, the phase of $q^{r}(s)$ automatically changes by $\pi$ between zero crossings of $\operatorname{Re}\left[q^{r}(s)\right]$. Thus as long as the phase of $q^{\mathrm{r}}(s)$ is kept continuous, taking a square root results in the proper number of $\pi / 2$ phase shifts.

\section{Example $G$}

In this example the effect of the scale of a velocity inhomogeneity on the Gaussian beam solution is investigated. A

\section{LAYER OVER GRADIENT}
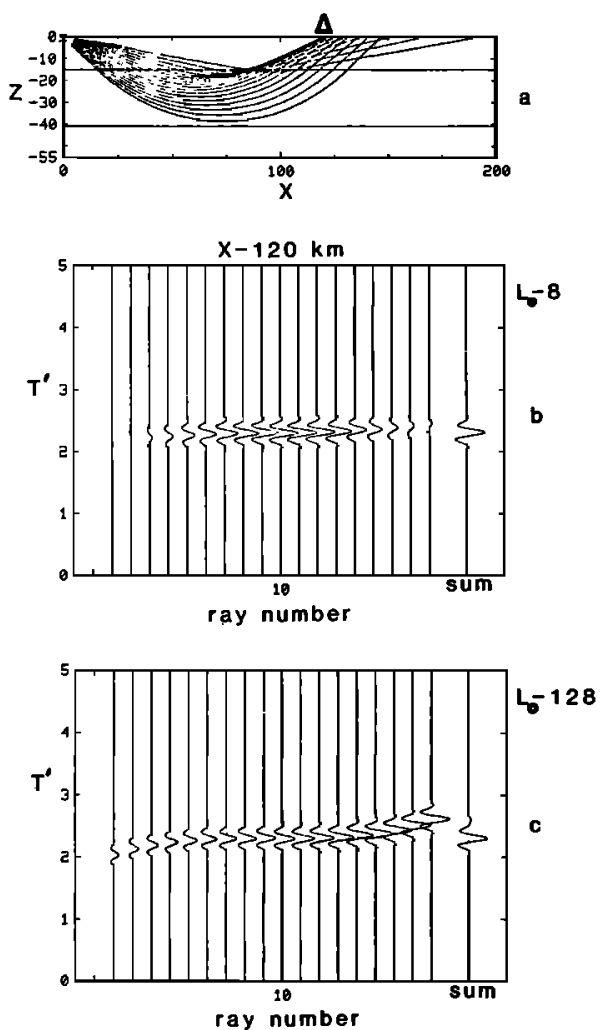

Fig. 10. Layer over gradient. (a) Ray diagram with station at $x=120 \mathrm{~km}$. (b) Individual beam contributions and resulting sum for $L_{0}=8 \mathrm{~km}^{1 / 2}$. (c) Individual beam contributions and resulting sum for $L_{0}=128 \mathrm{~km}^{\mathrm{i} / 2}$

\section{LAYER OVER GRADIENT}
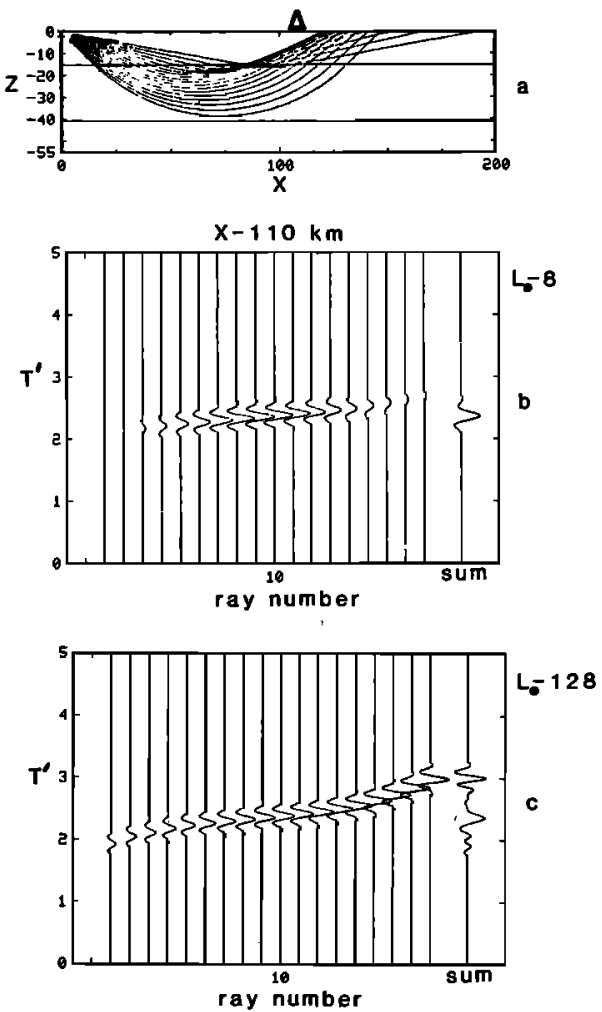

Fig. 11. Layer over a gradient. (a) Ray diagram with station at $x=110 \mathrm{~km}$. (b) Individual beam contributions and resulting sum for $L_{0}=8 \mathrm{~km}^{1 / 2}$. (c) Individual beam contributions and resulting sum for $L_{0}=128 \mathrm{~km}^{\mathrm{i} / 2}$
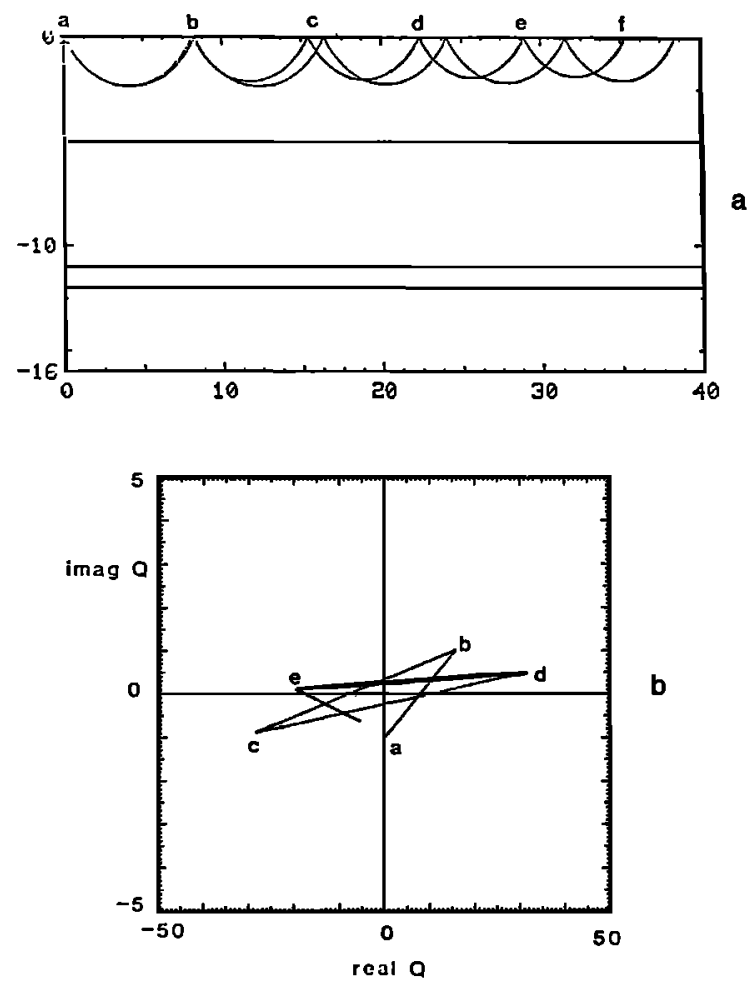

Fig. 12. Rays in a surface wave guide. (a) Ray diagram. (b) Graph of the complex function $q^{\mathrm{T}}(s)$. 


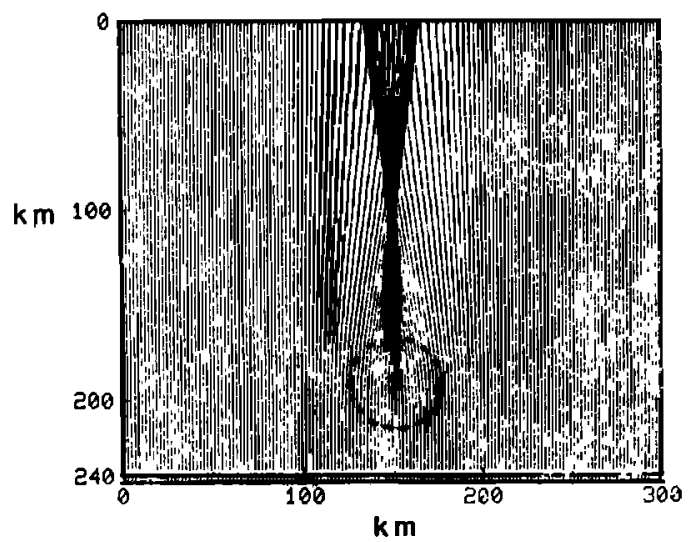

Fig. 13. Ray diagram for a single heterogeneity with a radius of 25 $\mathrm{km}$ and a velocity contrast of $10 \%$.

single heterogeneity is considered with an incident plane wave impinging from below. The background velocity is $6 \mathrm{~km} / \mathrm{s}$, and the wavelet parameters are $f=1 \mathrm{~Hz}, \gamma=4, \varphi_{0}=0$. The velocity heterogeneity is circular, and the velocity varies smoothly in a Gaussian manner. The radius of the heterogeneity ranges from 15 to $25 \mathrm{~km}$ with a velocity contrast of $10 \%$ lower than the background. The Gaussian beam solution is then compared with the finite difference solution of the $45^{\circ}$ parabolic equation typically used in seismic exploration and forward scattering simulations [see Claerbout, 1976; Kjartansson, 1980]. The ray diagram for a $25-\mathrm{km}$ radius heterogeneity is shown in Figure 13.

Figure 14 shows the seismograms for an inhomogeneity with a radius of $25 \mathrm{~km}$, a velocity contrast of $10 \%$, a distance from the initial wave front to the heterogeneity of $50 \mathrm{~km}$, and a distance from the initial wave front to the stations of 120 $\mathrm{km}$. The finite difference solution of the parabolic equation, Parab45, is shown in Figure $14 a$. Figures $14 b-14 d$ show the Gaussian beam solutions for three initial beam widths. The critical initial beam width for the station distance in a homogeneous medium is $L_{0_{\mathrm{r}}}=11 \mathrm{~km}^{1 / 2}$. From the results of example $A$, the decomposition of an initial plane wave in a homogeneous medium requires initial beam widths comparable with or smaller than the critical initial beam width for the receiver distance. The maximum amplitude for each solution is shown in Figure 14. The Gaussian beam results for the different initial beam widths are seen to be stable and compare with the Parab45 result.

Figure 14 also shows the beam width in kilometers at the inhomogeneity distance for each initial beam width solution. The smallest beam width at the heterogeneity distance is 14 $\mathrm{km}$, resulting from an initial beam width of $L_{0}=7 \mathrm{~km}^{1 / 2}$, and is smaller than the heterogeneity radius. From example $A$, this smallest beam width defines the zone of contributing beams to the final sum. For smaller than critical initial beam widths a similar limited region is defined by phase interference about the stationary direct arrival and is related to the first Fresnel zone.

Figure 15 shows the same experiment, except now the initial wavefront is $140 \mathrm{~km}$ from the heterogeneity and $210 \mathrm{~km}$ from the stations. The Parab45 result is shown in Figure 15a, and the Gaussian beam seismograms for four initial beam widths are shown in Figures $15 b-15 e$. Due to the spread of the beams with distance, the beam widths at the heterogeneity are larger

\section{SINGLE INHOMOGENEITY}

$$
\begin{aligned}
& a-25 \mathrm{~km} \mathrm{A1} \\
& v 6-.6 \mathrm{~km} / \mathrm{s} \theta \mathrm{C}
\end{aligned}
$$

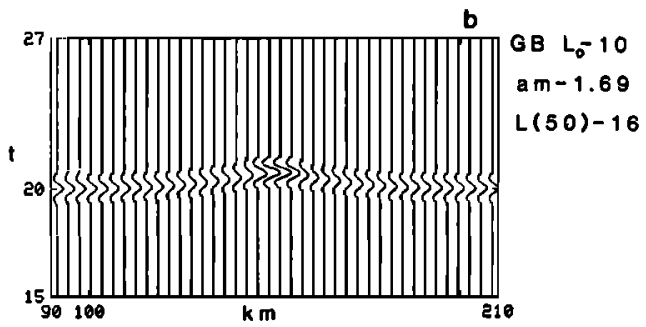

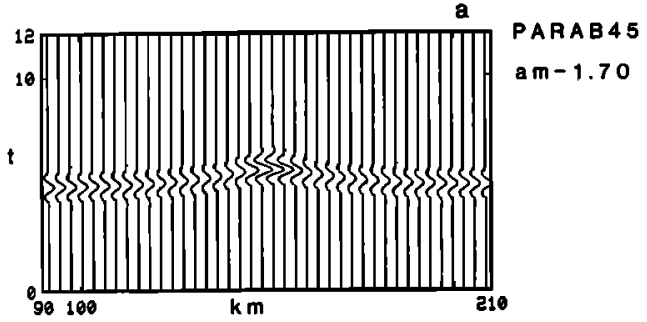
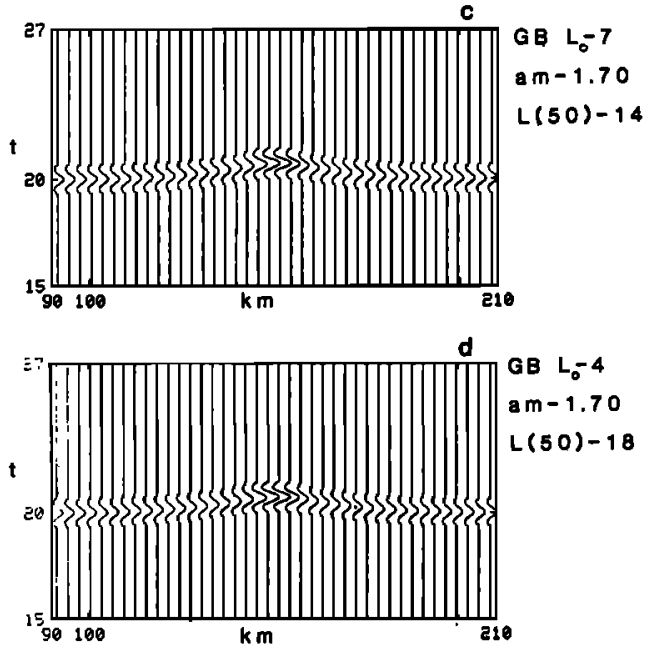

Fig. 14. Single velocity heterogeneity with a radius of $25 \mathrm{~km}$, a velocity contrast of $10 \%$, the distance from the initial wave front to the heterogeneity of $50 \mathrm{~km}$, and the distance from the initial wave front to the stations of $140 \mathrm{~km}$. (a) Parab45 seismograms. Gaussian beam seismograms for $(b) L_{0}=10 \mathrm{~km}^{1 / 2},(c) L_{0}=7 \mathrm{~km}^{1 / 2}$, and $(d) L_{0}=4 \mathrm{~km}^{1 / 2}$. 
SINGLE INHOMOGENEITY

$$
\begin{aligned}
& a-25 \mathrm{~km} \quad B 1 \\
& v 6-.6 \mathrm{~km} / \mathrm{sec}
\end{aligned}
$$

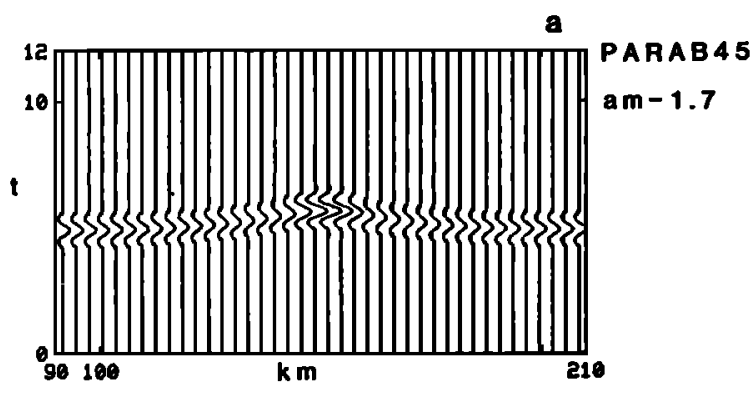

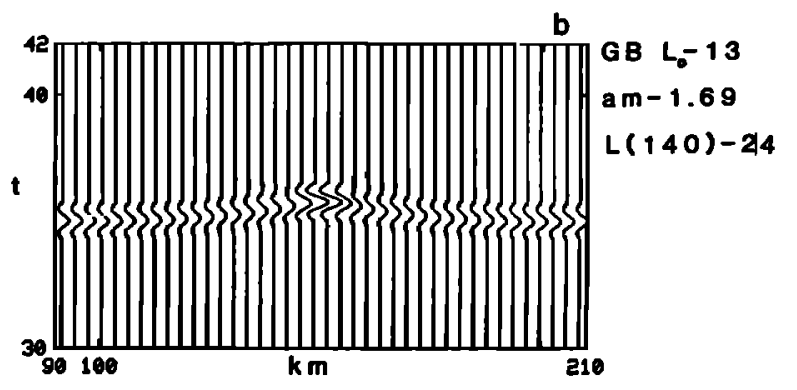

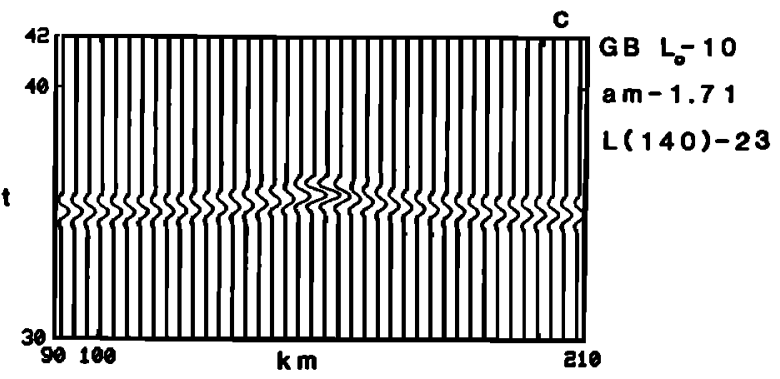

d

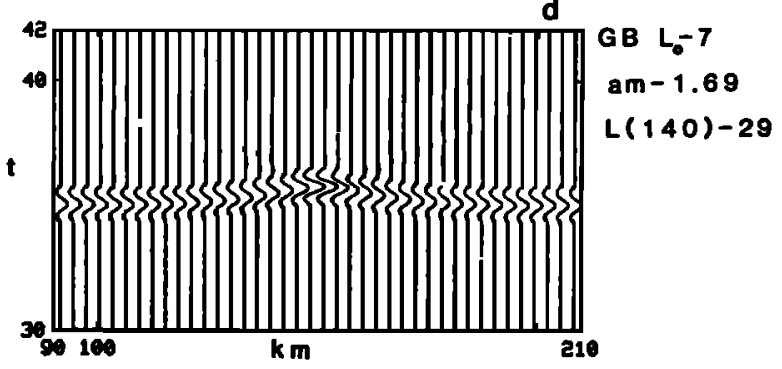

Fig. 15. Single velocity heterogeneity with a radius of $25 \mathrm{~km}$, a velocity contrast of $10 \%$, the distance from the initial wave front to the heterogeneity of $120 \mathrm{~km}$, and the distance from the initial wave front to the stations of $210 \mathrm{~km}$. (a) Parab45 seismograms. Gaussian beam seismograms for (b) $L_{0}=13 \mathrm{~km}^{1 / 2},(c) L_{0}=10 \mathrm{~km}^{1 / 2},(d) L_{0}=7 \mathrm{~km}^{1 / 2}$, and $(e)$ $L_{0}=4 \mathrm{~km}^{1 / 2}$.

than the previous case. The smallest beam width at the heterogeneity distance is $23 \mathrm{~km}$ and is just slightly smaller than the heterogeneity radius. The Gaussian beam results are still stable and compare with the Parab45 result. The $L_{0}=4 \mathrm{~km}^{1 / 2}$ result has a much larger beam width of $50 \mathrm{~km}$ at the heterogeneity distance, but the number of beams contributing is now limited by phase interference. The amplitude for this case is fairly stable, but a spurious secondary phase can be seen.

In the final case the inhomogeneity radius is reduced to 15 $\mathrm{km}$ with a velocity contrast of $10 \%$, a distance from the initial wave front to the heterogeneity of $30 \mathrm{~km}$, and a distance from the initial wave front to the stations of $210 \mathrm{~km}$. The Parab45 result is shown in Figure 16a. The Gaussian beam results are shown in Figures $16 b-16 e$ for several initial beam widths. For this case the Gaussian beam amplitudes are less stable. The Gaussian beam solution with the smallest beam width at the heterogeneity compares best with the Parab45 result. All the Gaussian beam solutions have a larger secondary arrival than the Parab45 solution. This may in part be due to dip filtering in the Parab45 formulation.

These preliminary results indicate that when using the Gaussian beam method in a heterogeneous medium, the critical beam width be smaller than the heterogeneity scale. This is more restrictive than simply small wavelength with respect to heterogeneity scale, and is related to the approximation made in the final term in (5) or (7). This restriction is similar to that given for geometric optics by Kravtsov and Orlov [1980] in which the medium and wave parameters must be slowly varying over the first Fresnel zone.

\section{Example $H$}

In this example, $S H$ waves in a two-dimensional basin structure are investigated. This problem has been studied by a number of investigators using techniques including finite difference, finite element, discrete wave number, and glorified optics (a ray method) [Boore et al., 1971; Hong and Helmberger, 1977; Bard and Bouchon, 1980; Aki and Richards, 1980]. The basin structure varies in thickness from $1 \mathrm{~km}$ at the edge to $6 \mathrm{~km}$ in the center over a range of $25 \mathrm{~km}$. The interface has a cosine shape. The material parameters in the basin are $v_{1}=$ $0.7 \mathrm{~km} / \mathrm{s}$ and $\rho_{1}=2.0 \mathrm{~g} / \mathrm{cm}^{3}$, and the material parameters of the basement are $v_{2}=3.5 \mathrm{~km} / \mathrm{s}$ and $\rho_{2}=3.3 \mathrm{~g} / \mathrm{cm}^{3}$.

Figure 17 shows the ray diagrams for the direct wave and the first two bounces. The rays represent an upward traveling $S H$ plane wave normally incident from below. The Gaussian beam synthesis was made up of 12 bounces. The incident plane wave was expressed by equally spaced rays in the basement. No two-point ray tracing was required; the rays were simply allowed to reverberate in the basin. For this case, 150 rays were used with a ray spacing of $1 \mathrm{~km}$. 
SINGLE INHOMOGENEITY

$$
\begin{gathered}
a-15 \mathrm{~km} \mathrm{A2} \\
v-6 .-.6 \mathrm{~km} / \mathrm{sec}
\end{gathered}
$$

a

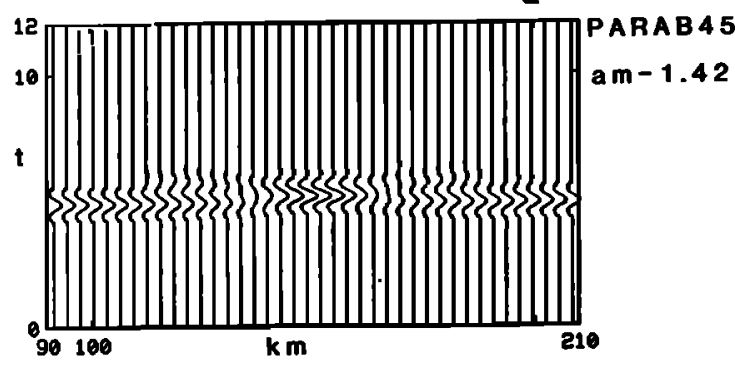

b

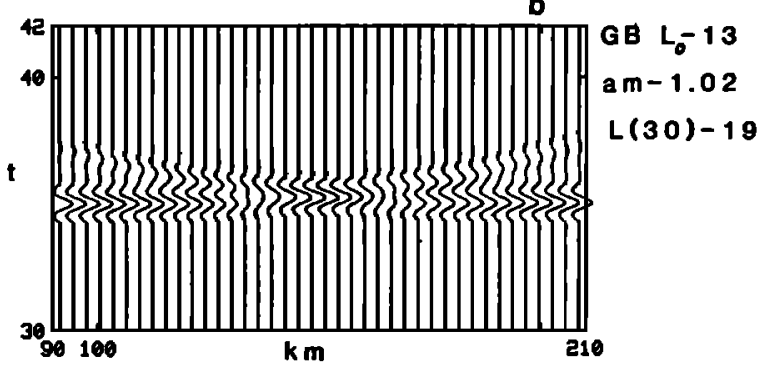

c

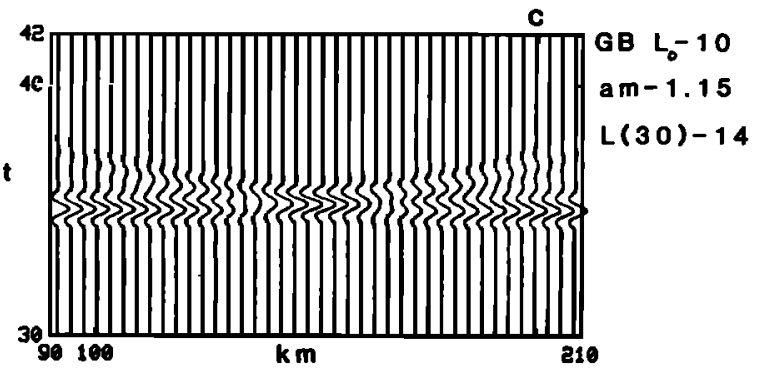

d

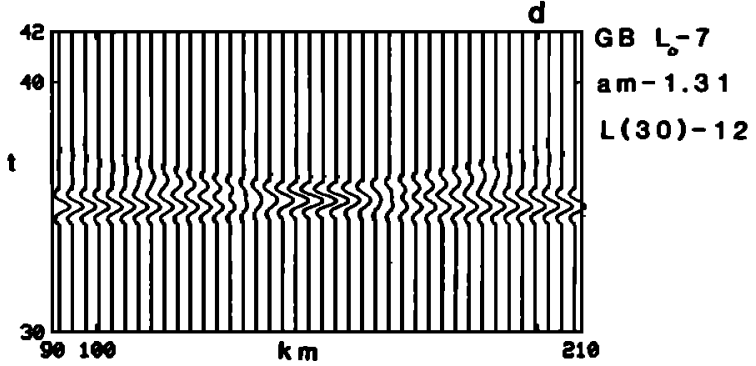

Fig. 16. Single velocity heterogeneity with a radius of $15 \mathrm{~km}$, a velocity contrast of $10 \%$, the distance from the initial wave front to the heterogeneity of $50 \mathrm{~km}$, and the distance from the initial wave front to the stations of $230 \mathrm{~km}$. (a) Parab45 seismograms. Gaussian beam seismograms for $(b) L_{0}=13 \mathrm{~km}^{1 / 2},(c) L_{0}=10 \mathrm{~km}^{1 / 2},(d) L_{0}=7 \mathrm{~km}^{1 / 2}$, and $(e)$ $L_{0}=4 \mathrm{~km}^{1 / 2}$.

The damped cosine source wavelet used in the Gaussian beam synthesis (see (21)) was adjusted to simulate an often used Ricker wavelet [see Bard and Bouchon, 1980]. In Figure 18 the solid line is the Ricker wavelet, and the dashed line is the damped cosine wavelet used in the Gaussian beam synthesis with $T=16.5 \mathrm{~s}$ and $\gamma=3.2$. The value of $L_{0}$ was set at $2.0 \mathrm{~km}^{1 / 2}$.

Figure 19 shows a comparison of four different methods for the two-dimensional basin structure. The Gaussian beam seismograms have been added to Figure 13.26 of Aki and Richards [1980]. The center of the basin is marked as $0 \mathrm{~km}$, and the edge of the basin as $20 \mathrm{~km}$. For each distance the Gaussian beam seismogram is at the bottom. The second phase on the seismograms represents energy propagating across the basin. The seismograms calculated by the Gaussian beam method compare well with the other solutions at the earlier portions of the seismograms. Discrepancies among the various methods are evident in the latter portions of the seismograms. This may be due to the fact that both the Gaussian beam method and glorified optics are high-frequency methods, while the discrete wave number method is applicable over a broader range of frequencies.

\section{Example I}

In this example the Gaussian beam method is applied to the study of volcanic earthquakes under Mount Saint Helens.
During the summer of 1981 a nine-station array was deployed on the flanks and within the crater of the Mount Saint Helens volcano. This was a joint effort undertaken by Oregon State University, Massachusetts Institute of Technology, and the U.S. Geological Survey [Fehler and Chouet, 1982]. Two of the more significant observations of the recorded high-frequency volcanic events were that (1) the arrival times at the crater station were between 0.4 and $0.7 \mathrm{~s}$ earlier than those at the flank stations and (2) the amplitudes at the crater station were about $\mathbf{1 0}$ times greater than those recorded at the flank station.

Figure 20 shows the two-dimensional model of the velocity structure that was tentatively adopted for Mount Saint Helens. This model is based on evidence obtained by various workers on Mount Saint Helens (S. Malone, lecture notes, 1982) as well as on other similar types of volcanoes such as Saint Augustine in Alaska [Kienle et al., 1979] and Showashinzan in Japan [Hayakawa, 1957]. In all these cases the internal structure of a volcano is depicted as a high-velocity central body with a $P$ velocity of about $4 \mathrm{~km} / \mathrm{s}$ covered by lower-velocity surficial layers.

It was first thought that the observed high amplitude and early arrival time could be explained by locating the earthquake foci at the bottom of the hypothesized high-velocity column. Figure 20 shows the ray diagram for a focus located $3.5 \mathrm{~km}$ below the summit. The high-velocity column tends to 
cause energy to diverge from the summit. Vertical component Gaussian beam seismograms for stations located across the volcano from 2.5 to $7.5 \mathrm{~km}$ along the surface are shown in Figure 21 using frequencies of 5,10 , and $20 \mathrm{~Hz}$. The amplitudes at the flank stations appear to be comparable to those at the crater, and the differences in arrival time are within $0.25 \mathrm{~s}$.

If the focal depth is moved upward to $1 \mathrm{~km}$ below the summit, the seismic signal changes dramatically. The ray diagram for a focus $1 \mathrm{~km}$ below the summit is shown in Figure 20 . In this case the ray paths going directly upward are dense and short, while those toward the flank of the volcano are sparse and take a detour. The resultant seismograms for 5, 10, and $20 \mathrm{~Hz}$ are shown in Figure 22. These show an amplitude at the crater station an order of magnitude greater than at the flank stations and about $0.5 \mathrm{~s}$ earlier arrival, in agreement with observation.

In order to check the accuracy of the computed seismograms, reciprocal seismograms were compared in Figure 23. In case A a two-dimensional vertical point force is applied at a point $3.5 \mathrm{~km}$ beneath the summit, and the receiver is on the flank of the volcano. In case B the vertical point force is on the flank of the volcano, and the receiver is located $3.5 \mathrm{~km}$ beneath the summit. The free surface is neglected here. The vertical component seismograms are shown, for both cases, for
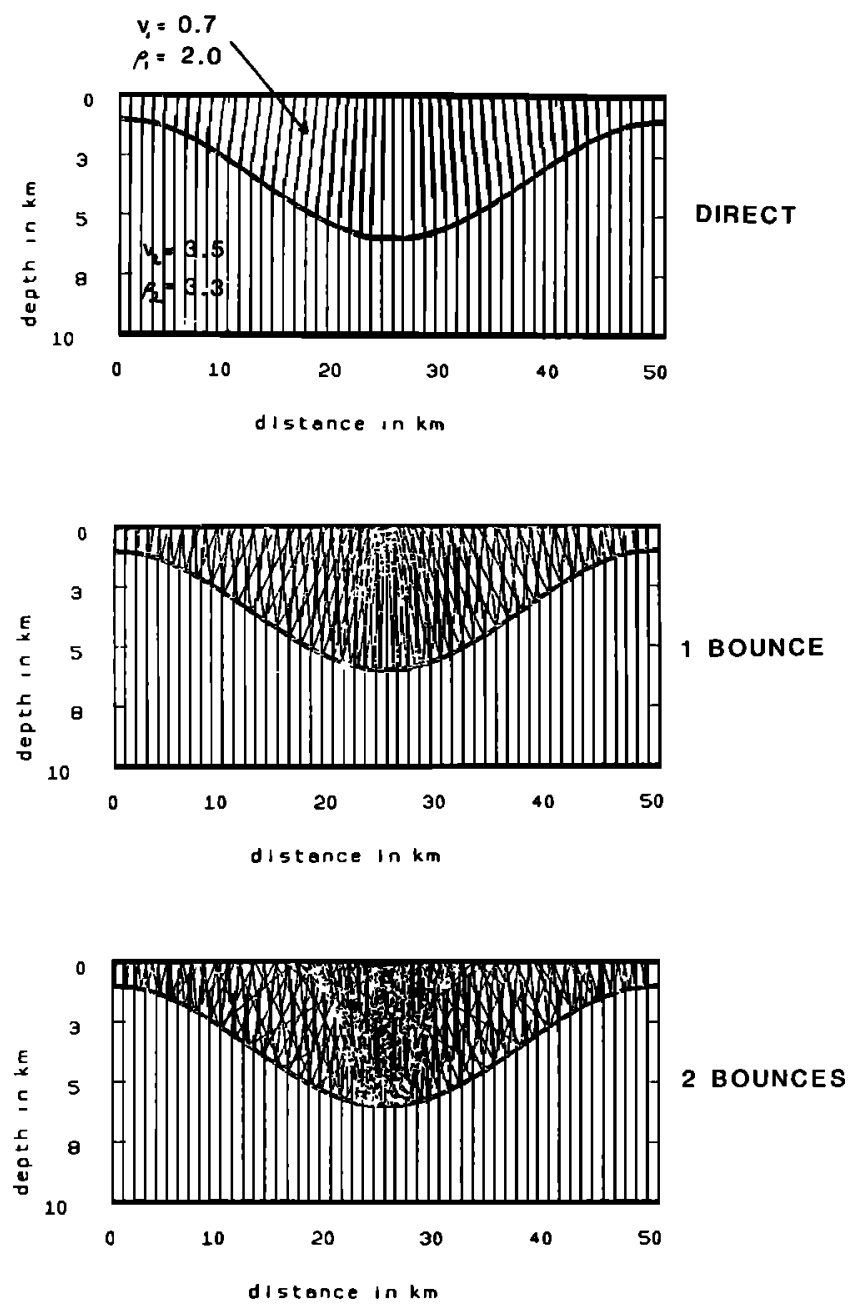

Fig. 17. Ray diagrams for an $S H$ plane wave incident on a twodimensional basin structure. The direct wave and first two bounces are shown.

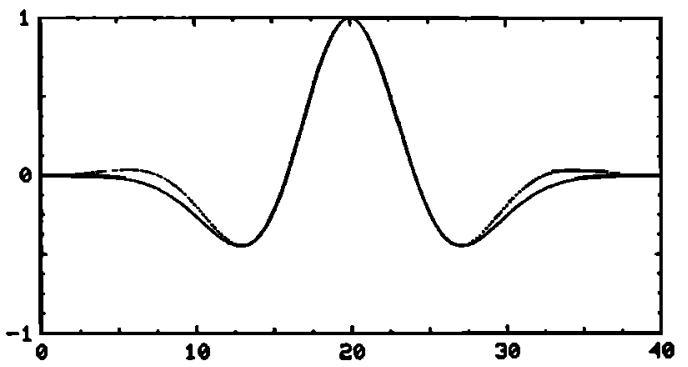

Fig. 18. Comparison between a Ricker wavelet (solid line) used in other studies of the soft basin, and the damped cosine wavelet (dotted line) used for the Gaussian beam seismograms with $T=16.5 \mathrm{~s}$ and $\gamma=3.2$.

frequencies of 5,10 , and $20 \mathrm{~Hz}$. There is a satisfactory agreement in wave form for all three frequencies. The amplitudes agree to within $1 \%$ for $20 \mathrm{~Hz}, 6 \%$ for $10 \mathrm{~Hz}$, and $12 \%$ for 5 $\mathrm{Hz}$, showing better accuracy for higher frequencies, as expected.

Thus the $P$ velocity structure depicted in Figure 20 and focal depths less than $1 \mathrm{~km}$ from the summit can explain the two significant observations. A similar observation was made for Saint Augustine volcano by Lalla and Kienle [1982], who found a correlation between the amplitude ratio of summit to

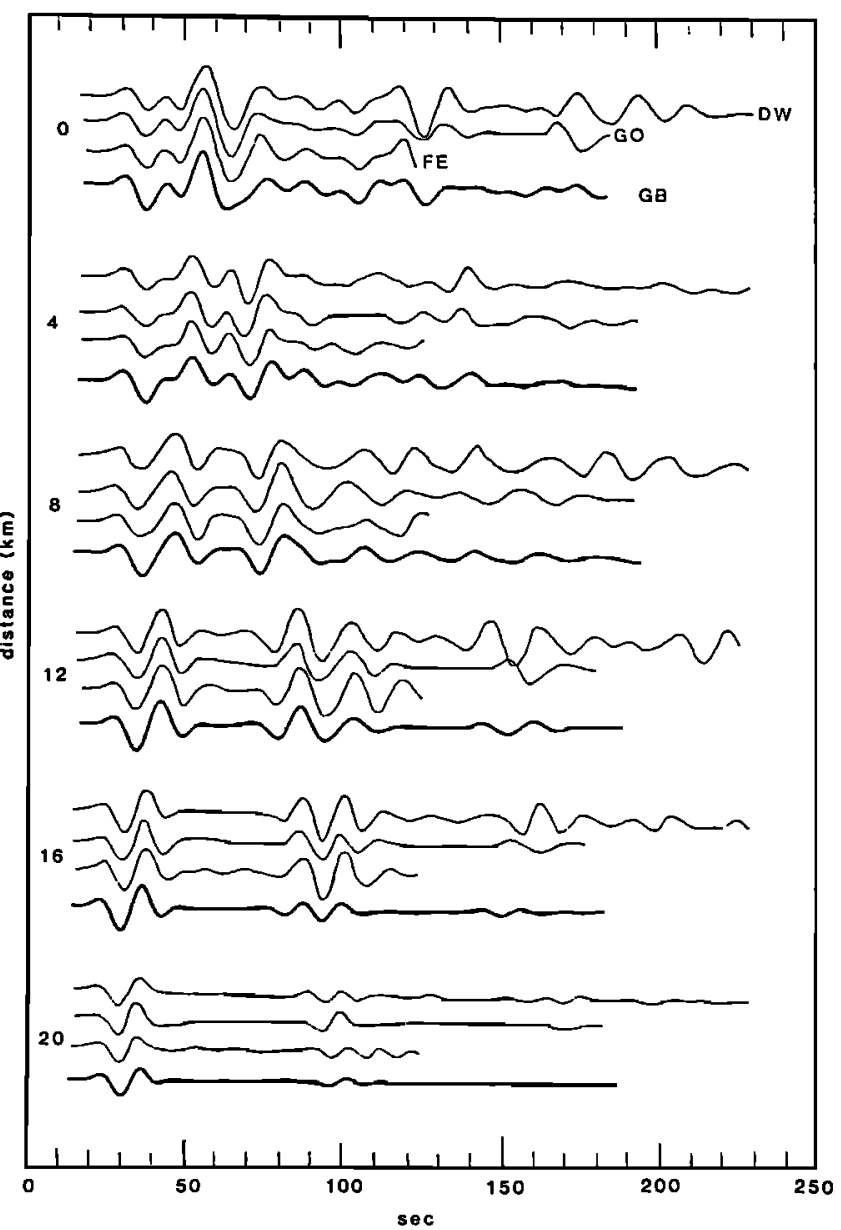

Fig. 19. Comparison of seismograms computed by various methods for a soft basin; DW discrete wave number, GO glorified optics (a ray method), FE finite element, and GB Gaussian beam. The center of the basin is marked as $0 \mathrm{~km}$, and the edge as $20 \mathrm{~km}$. 
flank stations and the focal depth. For focal depths shallower than about $2 \mathrm{~km}$ the summit amplitude was greater by an order of magnitude than the flank amplitude, and for deeper events the opposite was observed.

In any case the sensitivity of the seismic signal to the focal depth is strong, owing to the heterogeneous structure under a volcano. This result also suggests a promising way of accurately locating volcanic earthquakes using variations in amplitude as well as in arrival time.

\section{Example $J$}

In this final example the Gaussian beam method is used to study the influence of small-scale fluctuations on teleseismic $P$ wave forms. The models consist of several realizations of a lithosphere with randomly fluctuating velocities. The layer thickness is $120 \mathrm{~km}$ with a correlation length of $15 \mathrm{~km}$. The average velocity is $8 \mathrm{~km} / \mathrm{s}$, and the root mean square fluctuation is $3 \%$, roughly representing the results obtained for LASA [Aki, 1973; Capon, 1974].

Normally distributed random velocities were used to construct a two-dimensioinal velocity grid with a $15-\mathrm{km}$ spacing between mesh points. This was then interpolated using bicubic splines to give continuous velocities as well as first and second derivatives. The first realization for the randomly fluctuating velocities is shown in Figure 24. The top portion is a contour plot of the random velocities contoured at $0.1-\mathrm{km} / \mathrm{s}$ interval. The bottom portion is a printer plot of the random velocities, where each integer on the plot represents a $0.3-\mathrm{km} / \mathrm{s}$ velocity interval and the integer 4 represents the velocity interval 7.7 $8.0 \mathrm{~km} / \mathrm{s}$.

Figure 25 shows the ray diagram for a vertically incident plane wave perturbed by the random velocity layer. The
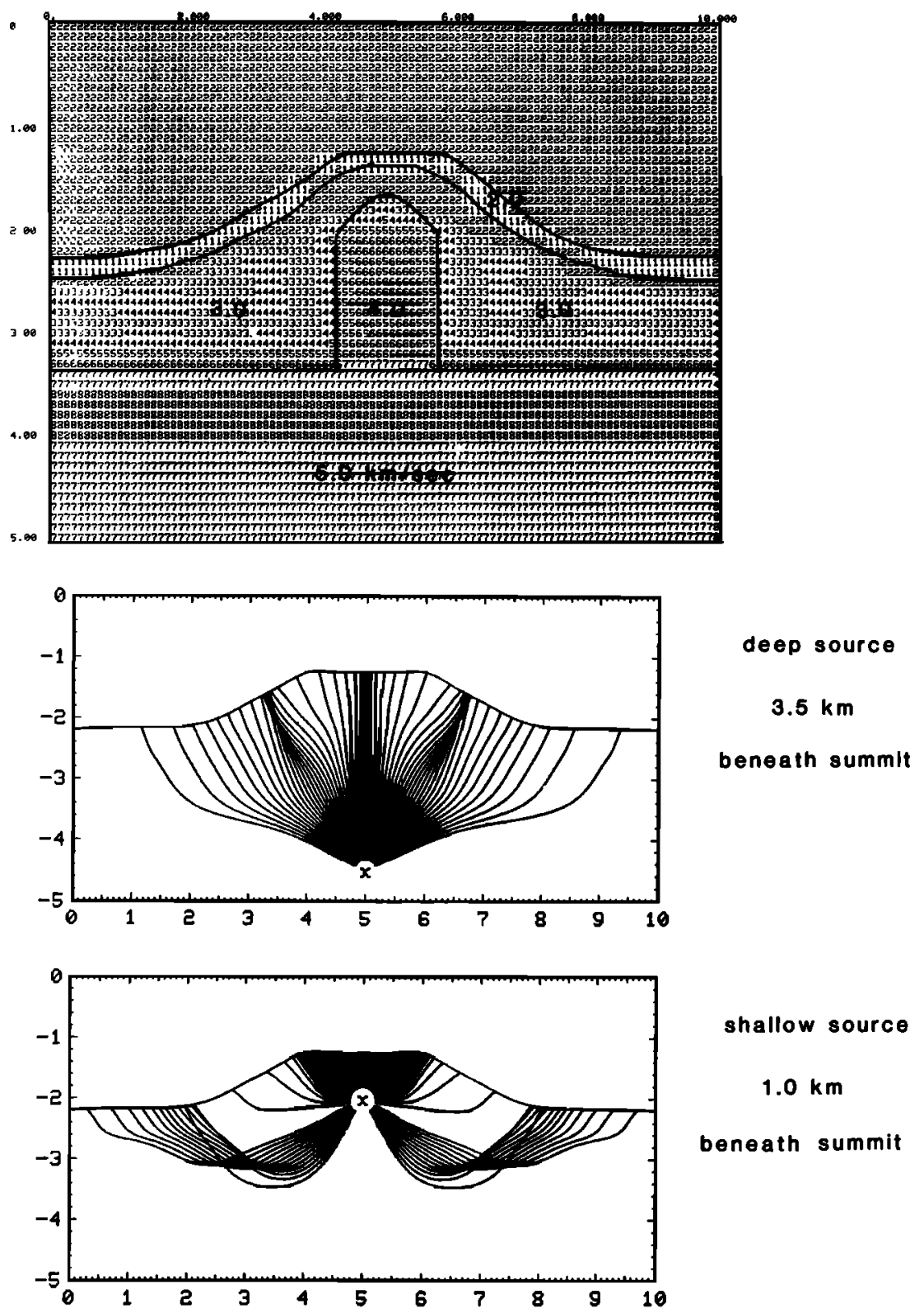

shallow source

$1.0 \mathrm{~km}$

beneath summit

Fig. 20. Velocity model used to depict the structure under Mount Saint Helens. Ray diagrams are shown below for a source located $3.5 \mathrm{~km}$ beneath the summit and a source located $1.0 \mathrm{~km}$ below the summit. 
receivers are located just above the random layer at $z=75$ $\mathrm{km}$. The range of receivers is from 125 to $275 \mathrm{~km}$, spaced at $5-\mathrm{km}$ intervals. Figure 26 shows the resulting wave field for two dominant frequencies, 2 and $1 \mathrm{~Hz}$. The amplitude and phase have both been significantly distorted by the random velocity layer. A multipath effect can also be seen in the range from 130 to $160 \mathrm{~km}$. No significant $P$ coda with a duration beyond about $3 \mathrm{~s}$ has developed as a result of the random velocity layer.

In order to check the accuracy of the method for this example, seismograms from reciprocal geometries have been compared using a two-dimensional point force as a source. The first comparison, shown in Figure 27, is for a source and receiver $300 \mathrm{~km}$ above and below the random velocity layer. In this geometry the rays for the reciprocal cases sample the same region of the random layer. There is a good agreement in wave shape between reciprocal cases. Also, the amplitudes agree within $5 \%$. For this case the parameter $S_{0}$ was specified to put the minimum beam width (beam waist) just before the ray entered the random layer, and $L_{0}$ was specified as 12 $\mathrm{km}^{1 / 2}$.

In addition to reciprocity checks, the calculation was repeated using the finite difference solution of the $45^{\circ}$ equation [Claerbout, 1976]. Figure 28 shows a comparison between the parabolic result, Parab45, and the Gaussian beam result for the $1-\mathrm{Hz}$ case. The results are similar in the significant details.
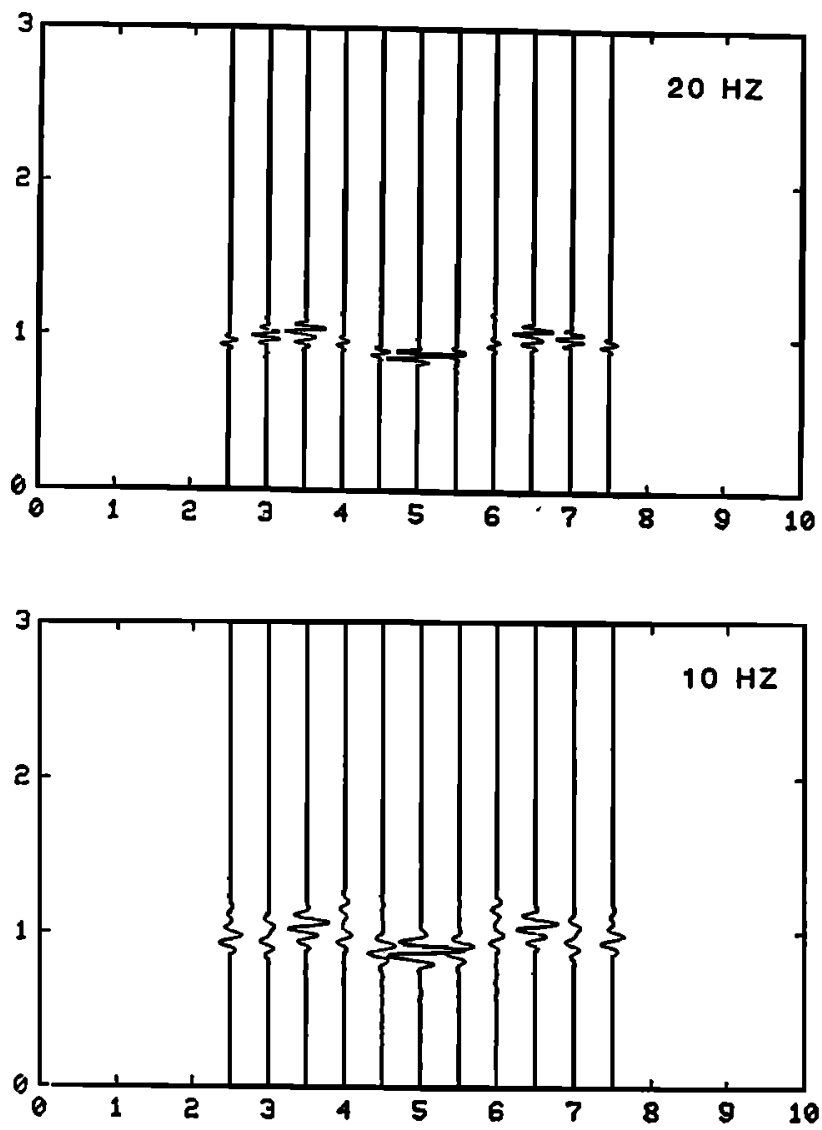

DEEP SOURCE

$(3.5 \mathrm{~km})$

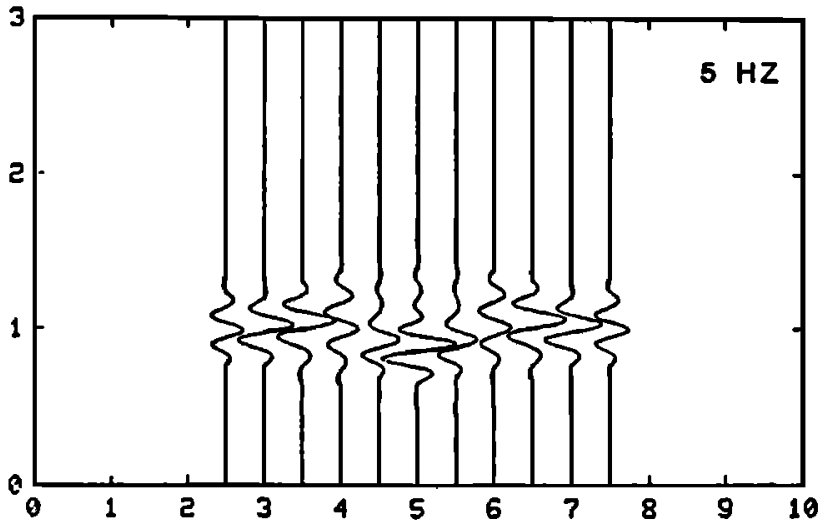

Fig. 21. Gaussian beam seismograms for a source located $3.5 \mathrm{~km}$ below the summit using three frequencies: 5,10 , and 20 
In Figure 29 a second realization for the random velocity layer is shown. The perturbed rays for an incident plane wave are shown in Figure 30. The wave fields observed on the surface of the random velocity layer for the two dominant frequencies of 2 and $1 \mathrm{~Hz}$ are shown in Figure 31. This realization perturbs the wave field less than the previous realization. The results have been checked using reciprocity and compared against the Parab45 solution and showed good agreement. For this realization a comparison has been made between the natural logarithm amplitude and the residual phase delay. This is shown in Figure 32. The positive correlation between $\ln (A)$ and the phase delay residual is in agreement with observations of teleseismic $P$ waves at the Montana LASA by $A k i$ [1973].

\section{Conclusions}

There are several advantages in using the Gaussian beam method. First, the method is always finite at caustics. In addition, no prior knowledge of caustic locations is required as in other methods. Next, since the Gaussian beam method relies on local parabolic approximations, no global direction of propagation must be assumed as in the standard parabolic approximation. Finally, the Gaussian beam method is comparable in cost to ray methods, and possibly faster since no two-point ray tracing is done.

The Gaussian beam result depends on the choice of the initial beam width. The expansion of a plane wave by Gaussian beams in a homogeneous medium requires initial beam
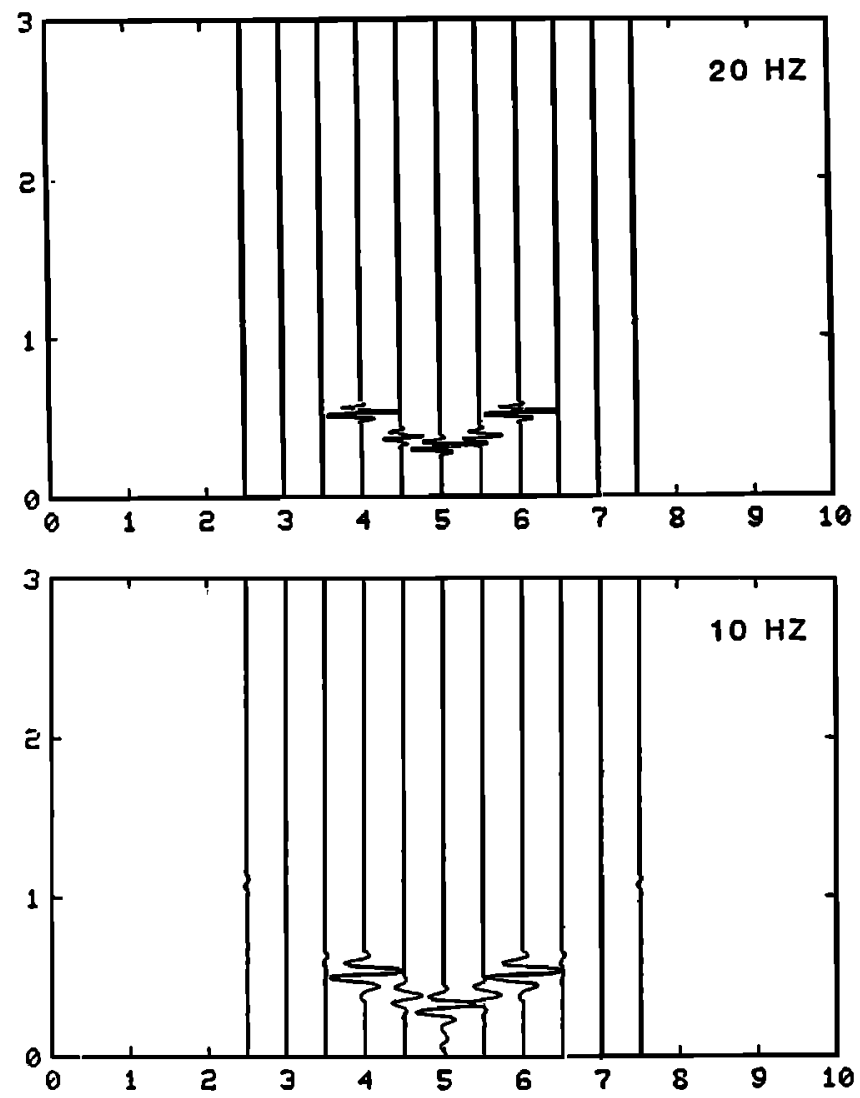

SHALLOW SOURCE

$(1.0 \mathrm{~km})$

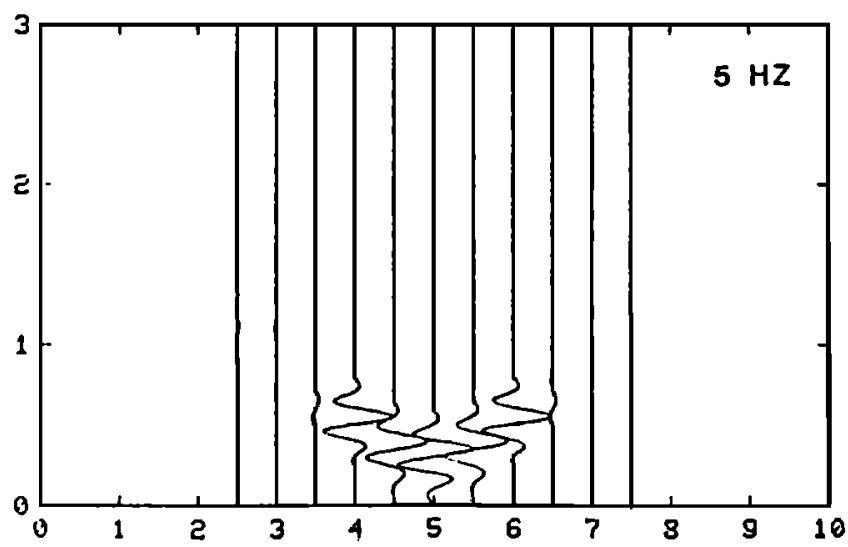

Fig. 22. Gaussian beam seismograms for a source located $1.0 \mathrm{~km}$ below the summit using three frequencies: 5,10 , and 20 


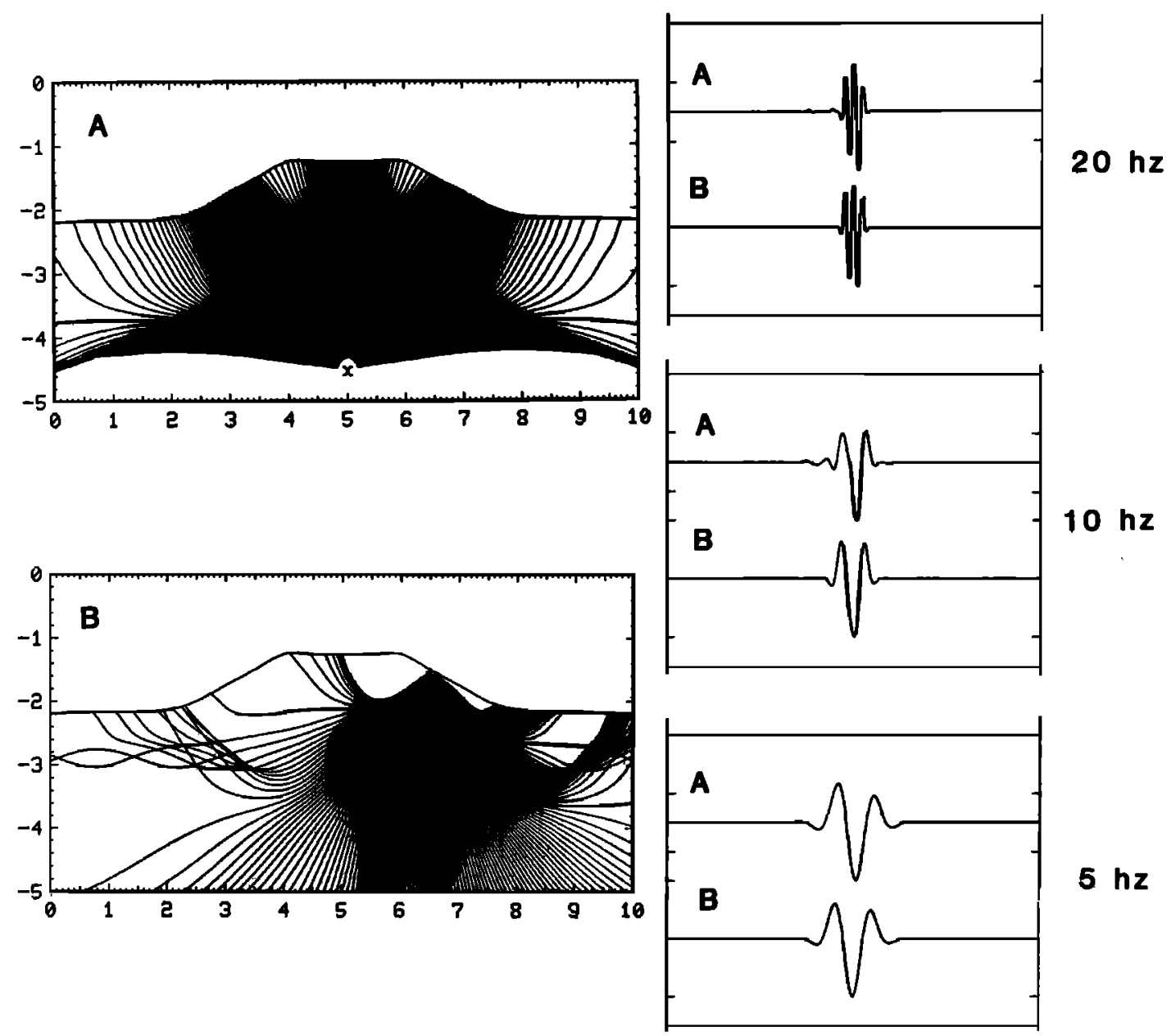

Fig. 23. Ray diagrams and seismograms shown for reciprocal cases A and B. The reciprocal seismograms are shown for three frequencies: 5,10 , and $20 \mathrm{~Hz}$.

widths comparable with or smaller than the critical initial beam width for a given station distance. This is similar to an expansion of an initial plane wave into pointlike sources as viewed from the station. The approximate expansion of a line source into Gaussian beams in a homogeneous medium requires initial beam widths comparable or larger than the critical initial beam width. This is similar to an expansion of a line source into plane waves. The use of initial beam widths near critical avoids end effects but does not simulate certain arrivals, such as head waves in the welded interface example.

The Gaussian beam method in a heterogeneous medium has been tested using two approaches. The first is the application of the reciprocal theorem, where the discrepancy between seismograms calculated for reciprocal cases is considered as a measure of error. The second is the application of the Gaussian beam method to cases, including the twodimensional basin problem, which have solutions from other approximate methods. Preliminary results for a single heterogeneity using the finite difference solution to the parabolic equation and the Gaussian beam method gave best comparisons for critical beam widths smaller than the heterogeneity scale.

The Gaussian beam method was then applied to the study of volcanic earthquakes under Mount Saint Helens. The observed differences in amplitude and arrival time between a station inside the crater and those at the flank can be explained by the combined effects of an anomalous velocity structure and a shallow focal depth. The method was also applied to study the influence of small-scale velocity fluctuations on teleseismic $\boldsymbol{P}$ wave forms. Significant amplitude and phase fluctuations due to a lithosphere with randomly fluctuating velocities were found, in agreement with observations by Aki [1973] at the Montana LASA.

\section{APPENDIX A}

In this appendix we give a derivation of the parabolic equation from the eikonal equation [see Gloge and Marcuse, 1969; and Marcuse, 1982]. By considering a variable end point in Fermat's integral, the eikonal or Hamilton-Jacobi equation can be obtained:

$$
|\nabla \tau|^{2}=v^{-2}
$$

The rays are the characteristics of this equation. In raycentered coordinates the eikonal equation can be written

$$
\frac{\partial \tau}{\partial s}-\frac{h_{1}}{v}\left[1-v^{2}\left(\frac{\partial \tau}{\partial n}\right)^{2}\right]^{1 / 2}=0
$$

where $h_{1}$ is the scale factor in (2). The Hamiltonian is written 
random velocity layer

$8 \mathrm{~km} / \mathrm{sec} \pm 3 \%, a=15 \mathrm{~km}$ iseed $=0$
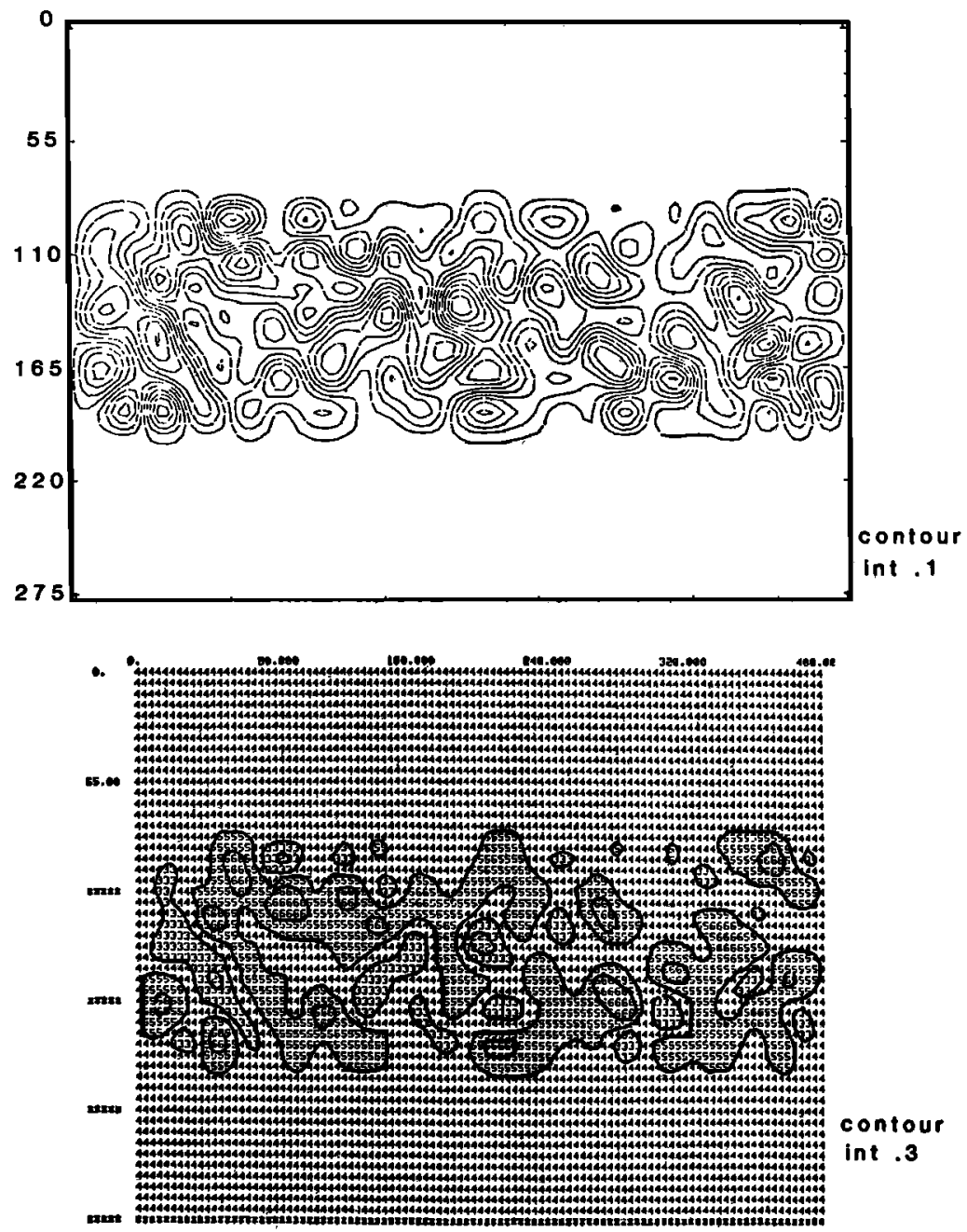

Fig. 24. Contour plot and printer plot of a layer with randomly fluctuating velocities. The average velocity is $8 \mathrm{~km} / \mathrm{s}$ with a root mean square fluctuation of $3 \%$. The correlation length is $15 \mathrm{~km}$. The contour interval is $0.1 \mathrm{~km} / \mathrm{s}$ for the contour plot, and $0.3 \mathrm{~km} / \mathrm{s}$ for the printer plot; random number seed is zero.

as

$$
H\left(n, p_{n}\right)=-\frac{h_{1}}{v}\left[1-v^{2} p_{n}^{2}\right]^{1 / 2}
$$

and $p_{n}=\partial \tau / \partial n$ is the slowness in the $n$ direction and is identified as the generalized momentum. The Hamiltonian can be approximated to second order about the central ray (see V. Cervený, unpublished lecture notes, 1982) as

$$
H\left(n, p_{n}\right)=-\frac{1}{v}+\frac{1}{2} v p_{n}^{2}+\frac{1}{2} v^{-2} v_{, n n} n^{2}
$$

Following Marcuse [1982], a parabolic equation can be derived by treating all variables as operators. By analogy to quantum mechanics,

$$
n \rightarrow n \quad p_{n} \rightarrow-i \kappa \frac{\partial}{\partial n} \quad H \rightarrow i \kappa \frac{\partial}{\partial s}
$$

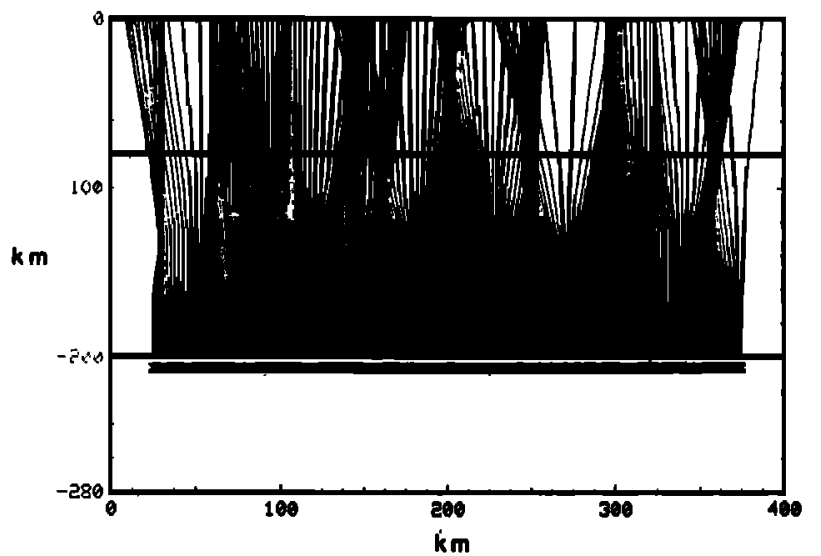

Fig. 25. Ray diagram of vertically incident plane wave perturbed by the random velocity layer shown in Figure 24. 

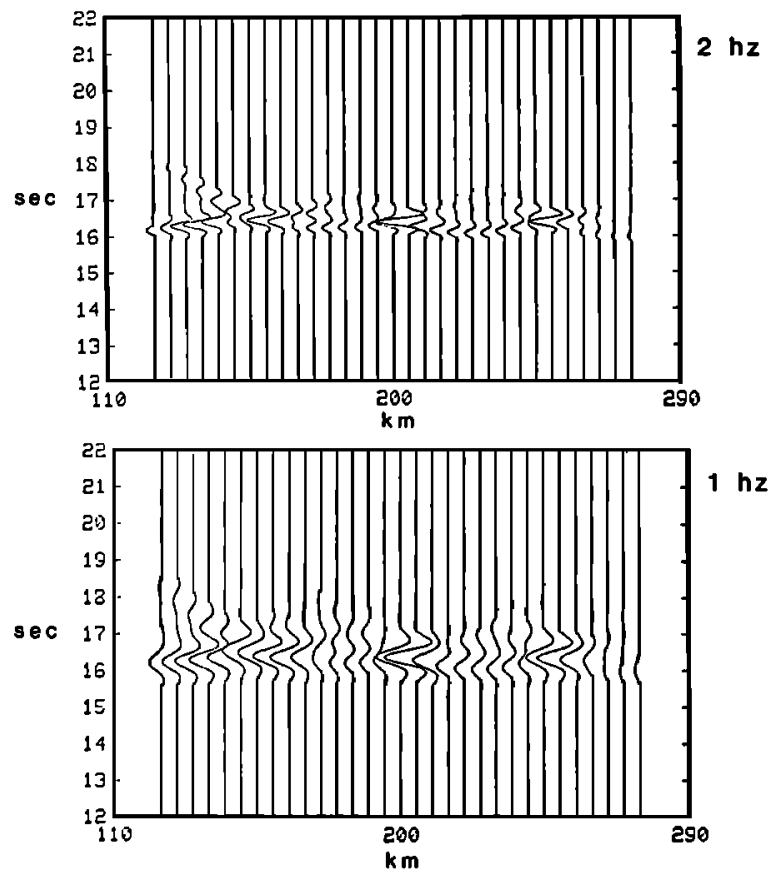

Fig. 26. Wave field of vertically incident plane wave propagated through random layer shown in Figure 24. Two frequencies are used: 1 and $2 \mathrm{~Hz}$.
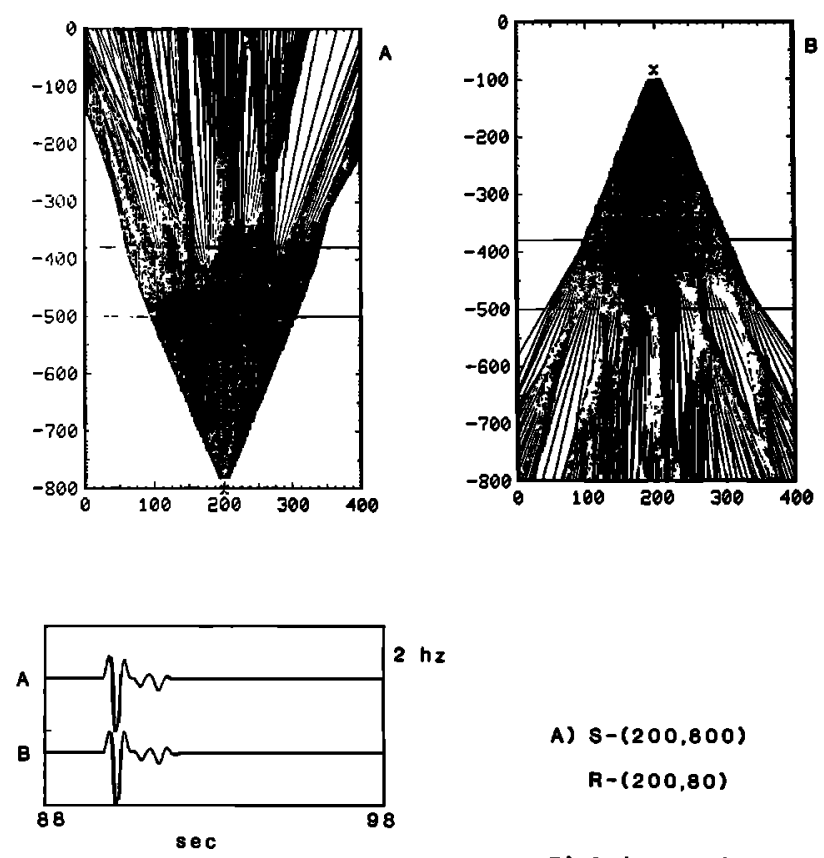

$$
\begin{aligned}
& \text { A) } s-(200,800) \\
& R-(200,80) \\
& \text { B) } s-(200,80) \\
& R-(200,800)
\end{aligned}
$$

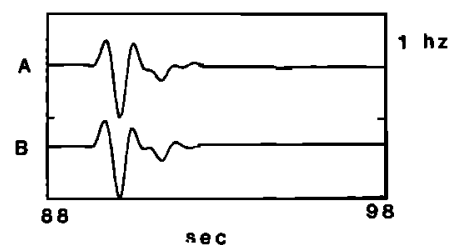

Fig. 27. Ray diagrams and seismograms for the shown reciprocal cases $A$ and $B$. For case $A$ the source is at $(200,800)$ and the receiver is at $(200,80)$. For case $B$ the source is at $(200,80)$ and the receiver at $(200,800)$. The random layer velocities are shown in Figure 24. The seismograms are computed for two frequencies: 1 and $2 \mathrm{~Hz}$.

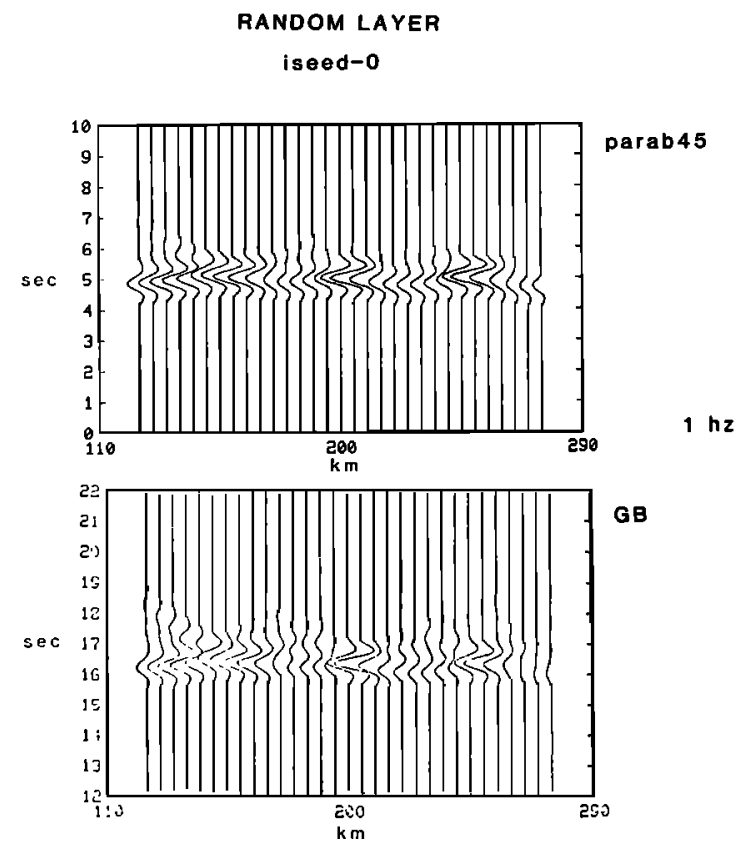

Fig. 28. Comparison between the Parab45 solution and the Gaussian beam solution for the random layer shown in Figure 24.

where $\kappa$ is a parameter to be determined. Applying the operator $\boldsymbol{H}$ to a wave function $\psi$,

$$
i \kappa \frac{\partial}{\partial s} \psi=H \psi
$$

Using equations (A4) and (A5), this is written

$$
\frac{2 i}{v \kappa} \psi_{, s}+\psi_{, n n}-\frac{1}{\kappa^{2} v^{3}} v_{, n n} n^{2} \psi+\frac{2}{v^{2} \kappa^{2}} \psi=0
$$

Writing $\psi$ in terms of the envelope

$$
\psi=W \exp \left[-i \omega\left(t-\int_{0}^{s} \frac{d s}{v(s)}\right)\right]
$$

and assuming $v=\omega^{1 / 2} n$, where $v=O(1)$, gives

$$
\begin{aligned}
\frac{2 i}{\kappa \omega v(s)} W_{, s}+W_{, v v}-\frac{1}{\kappa^{2} \omega^{2} v(s)^{3}} v_{, n n} v^{2} W \\
+\left(\frac{2}{\kappa^{2} \omega v(s)^{2}}-\frac{2}{\kappa v(s)^{2}}\right) W=0
\end{aligned}
$$

Letting $\kappa=\omega^{-1}$ gives

$$
\frac{2 i}{v(s)} W_{, s}+W_{, v v}-v(s)^{-3} v_{, n n} v^{2} W=0
$$

This is equivalent to the parabolic equation in (7) derived from asymptotic analysis. Note that in quantum mechanics $\kappa=h / 2 \pi$, where $h$ is Planck's constant, but here $\kappa=v_{0}\left(\lambda_{0} / 2 \pi\right)$. The $v_{0}$ can be avoided here by writing the eikonal equation (A2) in terms of $\tau^{\prime}=v_{0} \tau$. Classical ray theory is valid when $\lambda_{0}$ is much smaller than the heterogeneity scale. The natural analogy between mechanics and ray therory thus provides a link between the eikonal equation and the parabolic equation. Further results from this analogy can be found by Marcuse [1982] and Marcuvitz [1980]. 
$8 \mathrm{~km} / \mathrm{sec} \star 3 \%, a=15 \mathrm{~km}$ iseed -6

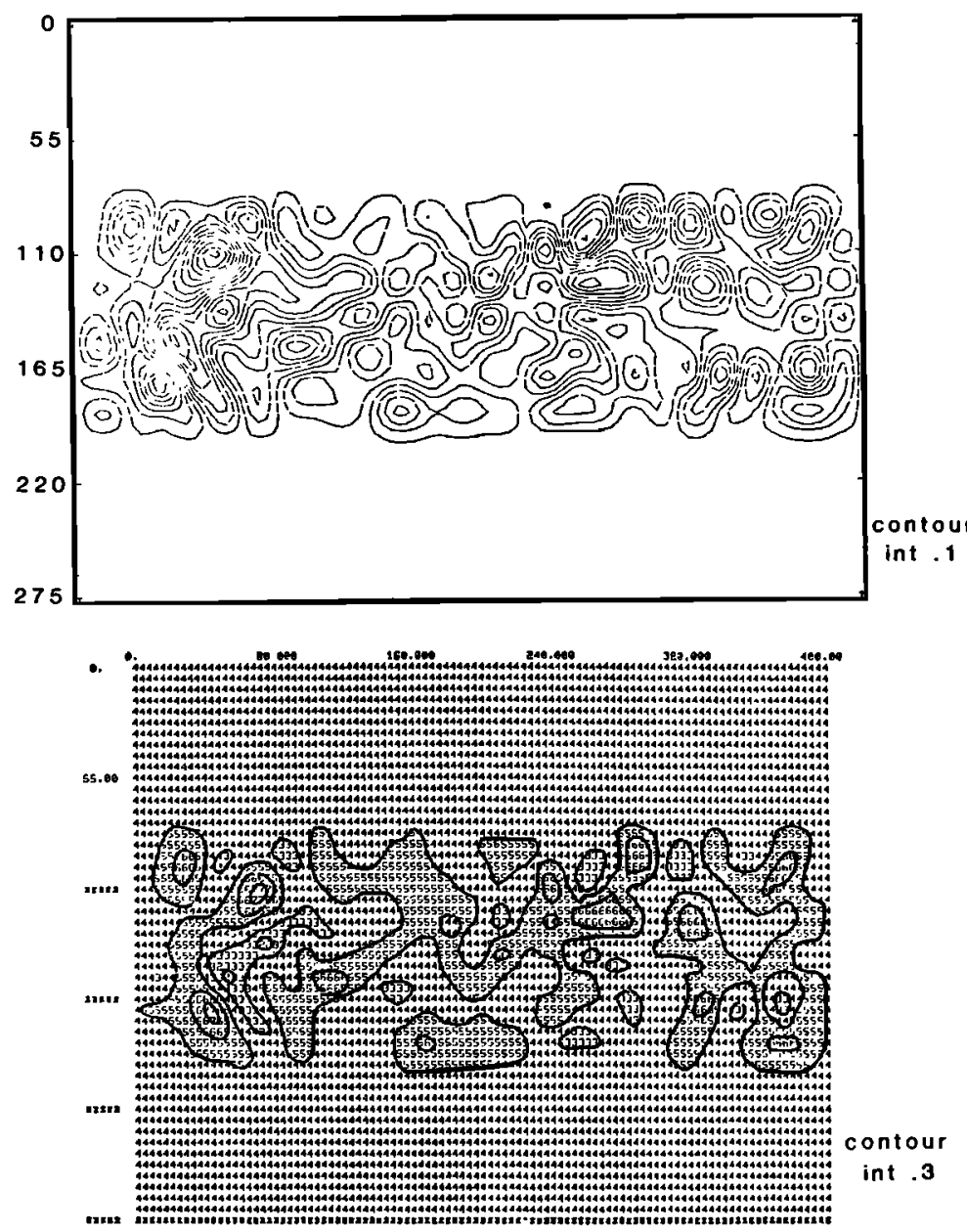

Fig. 29. Contour plot and printer plot of a layer with randomly fluctuating velocities. The average velocity is $8 \mathrm{~km} / \mathrm{s}$ with a root mean square fluctuation of $3 \%$. The correlation length is $15 \mathrm{~km}$. The contour interval is $0.1 \mathrm{~km} / \mathrm{s}$ for the contour plot and $0.3 \mathrm{~km} / \mathrm{s}$ for the printer plot; random number seed is 6 .

\section{APPENDIX B}

In this appendix a brief review of the properties of a Gaussian beam in a homogeneous medium is given. From the dynamic ray tracing equations (10) and the initial conditions for

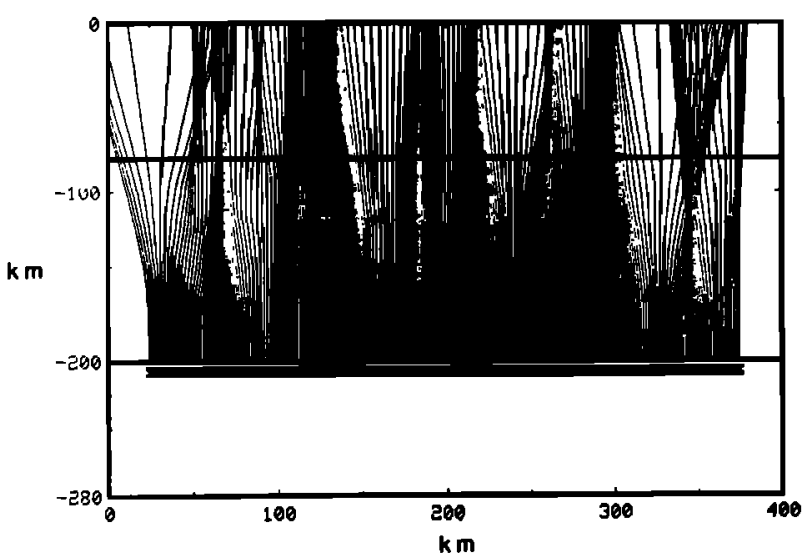

Fig. 30. Ray diagram of vertically incident plane wave perturbed by the random velocity layer shown in Figure 29. a plane wave and line source, one obtains

$$
\begin{array}{ll}
q_{1}(s)=1 & p_{1}(s)=0 \\
q_{2}(s)=s-s_{0} & p_{2}(s)=1 / v_{0}
\end{array}
$$

For the source at $s_{0}=0$, with the beam waist at the source, $S_{0}=0$, and using (16), the complex spreading can be written

$$
q(s)=s-i \frac{\omega}{2 v} L_{M}^{2}
$$

where $L_{M}$ is the initial beam width at the beam waist. The beam width can be written from (17) as

$$
L(s)=L_{M}\left[1+\left(\frac{\lambda s}{\pi L_{M}^{2}}\right)^{2}\right]^{1 / 2}
$$

The phase front curvature can be written from (18) as

$$
K(s)=\frac{1}{s\left[1+\left(\pi L_{M}{ }^{2} / \lambda s\right)^{2}\right]}
$$

At the beam waist, $L(s)=L_{M}$ and $K(s)=0.0$. Thus at the beam waist the minimum beam width is acheived and the wave front is planar. As $|s| \rightarrow \infty$, the angular spread or the 

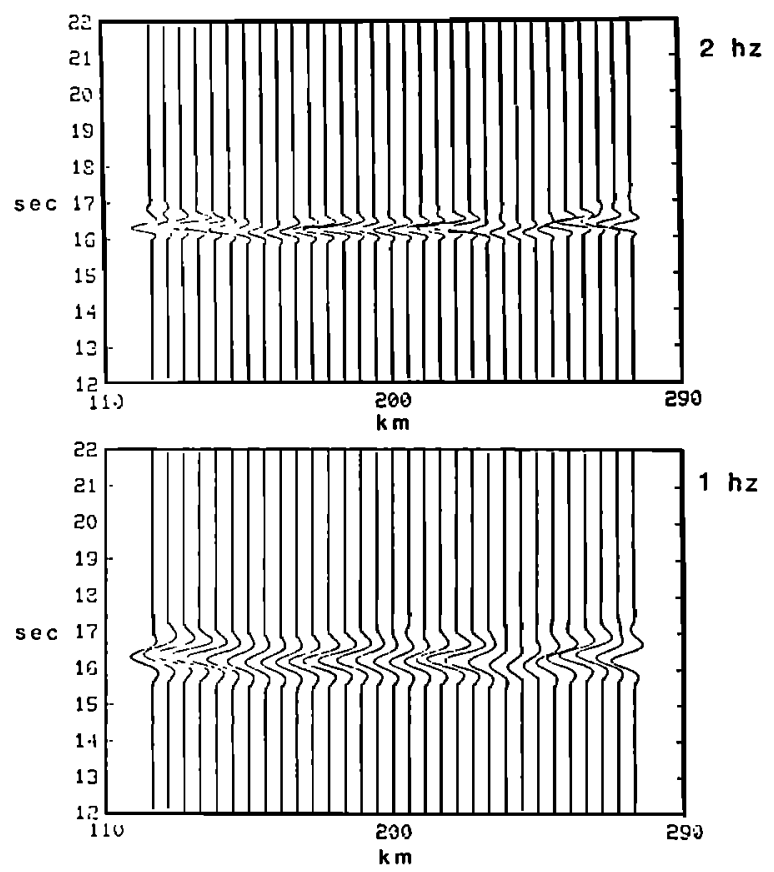

Fig. 31. Wave field of vertically incident plane wave propagated through random layer shown in Figure 29. Two frequencies are used: 1 and $2 \mathrm{~Hz}$.

far-field diffraction angle can be defined as

$$
\varphi=\lim _{|s| \rightarrow \infty}\left[\frac{L(s)}{s}\right]=\frac{\lambda}{\pi L_{M}}
$$

This leads to the uncertainty relation

$$
\varphi L_{M}=\lambda / \pi
$$

For a small $L_{M}$ there is a large far-field spread, whereas for a large $L_{M}$ there is a small far-field spread. The distance $s_{c}$, at which the beam width starts to significantly differ from the initial beam width, is

$$
\varphi \approx \frac{L_{M}}{s_{c}} \rightarrow s_{c}=\frac{\pi L_{M}^{2}}{\lambda}
$$

This is similar to the Fraunhofer range for an aperture of radius $a$, which has the form $s \gg a^{2} / \lambda$. For $|s| \gg s_{c}, K(s) \approx 1 / s$, which is the curvature of the wave front for a line source. Extrema in the curvature occur at $|s|=s_{c}$.

The critical value of $L_{M}$ is the one that gives the smallest beam width $L(s)$ at the receiver distance $s$. In a homogeneous medium this places the receiver distance at $s=s_{c}$. Thus from (B6), $L_{M_{\mathrm{cr}}}=(\lambda s / \pi)^{1 / 2}$.

\section{APPENDIX C}

In this appendix the weight function $\Phi(\delta)$ for a twodimensional point force in an elastic medium is obtained by comparing the Gaussian beam synthesis with the exact plane wave decomposition. For a general point force at the origin in the $x_{3}$ direction, the displacement field for an isotropic, homogeneous medium is given by

$$
\begin{aligned}
U_{i}= & \frac{1}{4 \pi \rho}\left[\left(3 \gamma_{i} \gamma_{3}-\delta_{i 3}\right)\left(\frac{r}{i \omega \beta}+\frac{1}{\omega^{2}}\right) r^{-3}\right. \\
& \left.-\left(\gamma_{i} \gamma_{3}-\delta_{i 3}\right)\left(\beta^{2} r\right)^{-1}\right] \exp \left(i \omega \frac{r}{\beta}\right) \\
& -\frac{1}{4 \pi \rho}\left[\left(3 \gamma_{i} \gamma_{s}-\delta_{i 3}\right)\left(\frac{r}{i \omega \alpha}+\frac{1}{\omega^{2}}\right) r^{-3}\right. \\
& \left.-\gamma_{i} \gamma_{3}\left(\alpha^{2} r\right)^{-1}\right] \exp \left(i \omega \frac{r}{\alpha}\right)
\end{aligned}
$$

[Aki and Richards, 1980], where a time dependence of $e^{-i \omega t}$ is assumed, and the direction cosines of $\mathbf{r}$ are given by $\gamma_{i}=$ $x_{i} / r$. In order to express the above displacements as an integral superposition of plane waves, the Weyl integral is used to obtain

$$
\begin{aligned}
& \frac{\partial}{\partial x_{3}} \frac{\partial}{\partial x_{i}}\left[\exp \left(i \omega \frac{r}{c}\right) / r\right] \\
& \quad=-\frac{i}{2 \pi} \iint_{-\infty}^{\infty} k_{i} \exp \left(i k_{1} x_{1}+i k_{2} x_{2}+i k_{c} x_{3}\right) d k_{1} d k_{2} \\
& \quad=\omega^{2}\left[\left(3 \gamma_{i} \gamma_{3}-\delta_{i 3}\right)\left(\frac{r}{i \omega c}+\frac{1}{\omega^{2}}\right) r^{-3}-\gamma_{i} \gamma_{3}\left(c^{2} r\right)^{-1}\right] \exp i \omega \frac{r}{c}
\end{aligned}
$$

where $k_{c}=+\left[\left(\omega^{2} / c^{2}\right)-k_{1}{ }^{2}-k_{2}{ }^{2}\right]^{1 / 2}$ and $c$ is either $\alpha$ or $\beta$.
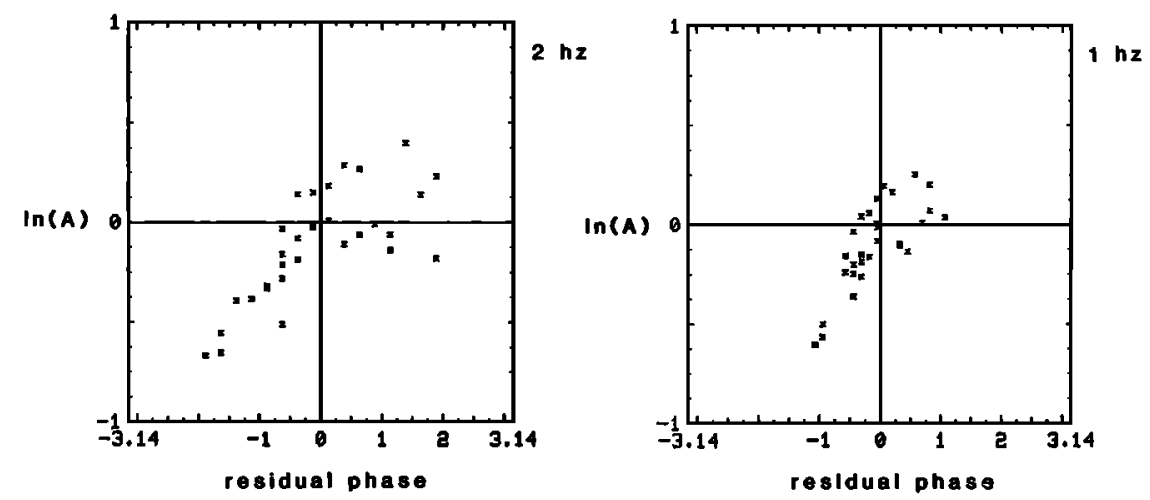

Fig. 32. Plots of $\ln$ (amplitude) versus residual phase delay for the wave fields shown in Figure 29. 
Then for the three-dimensional point force

$$
\begin{aligned}
U_{i}= & \frac{i}{8 \pi^{2} \rho \omega^{2}} \iint_{-\infty}^{\infty} k_{i} \\
& \cdot \exp \left[i\left(k_{1} x_{1}+k_{2} x_{2}+k_{\alpha} x_{3}\right)\right] d k_{1} d k_{2} \\
& -\frac{i}{8 \pi^{2} \rho \omega^{2}} \iint_{-\infty}^{\infty}\left[k_{i}-\frac{\delta_{i 3} \omega^{2}}{k_{\beta} \beta^{2}}\right] \\
& \cdot \exp \left[i\left(k_{1} x_{1}+k_{2} x_{2}+k_{\beta} x_{3}\right)\right] d k_{1} d k_{2}
\end{aligned}
$$

Now we will investigate the $P$ wave integral alone and integrate with respect to $x_{2}$ to obtain the line force solution. Thus

$$
\begin{aligned}
U_{L i} & =\int_{-\infty}^{\infty} U_{i} d x_{2} \\
& =\frac{i}{4 \pi \rho \omega^{2}} \int_{-\infty}^{\infty} k_{i} \exp \left[i\left(k_{1} x_{1}+k_{a} x_{3}\right)\right] d k_{1}
\end{aligned}
$$

where $k_{a}=k_{3}=+\left[(\omega / \alpha)^{2}-k_{1}{ }^{2}\right]^{1 / 2}$. Let $\vartheta$ be the angle with the $x_{3}$ axis, then $k_{1}=(\omega / \alpha) \sin \vartheta$ and $k_{3}=(\omega / \alpha) \cos \vartheta$. Now, writing the integral over $\vartheta$,

$$
\begin{gathered}
U_{L 1}=\int_{-\omega / \alpha}^{+\omega / \alpha} U_{1} d x_{2}=\int_{-\pi / 2}^{+\pi / 2} \frac{i}{4 \pi \rho \alpha^{2}} \cos \vartheta \sin \vartheta \\
\cdot \exp \left[i \frac{\omega}{\alpha}\left(x_{1} \sin \vartheta+x_{3} \cos \vartheta\right)\right] d \vartheta \\
U_{L 3}=\int_{-\omega / \alpha}^{+\omega / \alpha} U_{2} d x_{2}=\int_{-\pi / 2}^{+\pi / 2} \frac{i}{4 \pi \rho \alpha^{2}} \cos \vartheta \cos \vartheta \\
\cdot \exp \left[i \frac{\omega}{\alpha}\left(x_{1} \sin \vartheta+x_{3} \cos \vartheta\right)\right] d \vartheta
\end{gathered}
$$

The Gaussian beam solution for a homogeneous medium is written

$$
\begin{gathered}
U_{G B}(\mathbf{x}, \omega)=\int_{-\pi / 2}^{+\pi / 2} \Phi(\delta) u_{\delta}(s, n, \omega) d \delta \\
U_{G B}(\mathbf{x}, \omega)=\int_{-\pi / 2}^{+\pi / 2} \Phi^{\prime}(\delta)\left(\frac{\alpha_{0} \rho_{0} q_{0}}{\alpha \rho q}\right)^{1 / 2} \\
\cdot \exp \left[i \omega \frac{r}{\alpha}+i \frac{\omega}{2} \frac{p}{q} n^{2}\right] d \delta
\end{gathered}
$$

where it has been assumed that $\Phi(\delta)$ has the form, $\Phi(\delta)=$ $\Phi^{\prime}(\delta)\left(\alpha_{0} \rho_{0} q_{0}\right)^{1 / 2}$. For a homogeneous medium,

$$
\begin{array}{cc}
q=r+\varepsilon & q_{0}=\varepsilon \\
p=v_{0}{ }^{-1} & p_{0}=v_{0}^{-1} \\
p / q=\frac{1}{v_{0}[r+\varepsilon]}
\end{array}
$$

where $\varepsilon=S_{0}-i\left(\omega / 2 v_{0}\right) L_{M}{ }^{2}$. To compare with the plane wave solution, let $L_{M} \rightarrow \infty$ and let $\delta=9$. Then

$$
\begin{aligned}
& U_{G B}(\mathbf{x}, \omega)=\int_{-\pi / 2}^{\pi / 2} \Phi^{\prime}(\vartheta) \\
& \cdot \exp \left[i \frac{\omega}{\alpha}\left(x_{1} \sin \vartheta+x_{3} \cos \vartheta\right)\right] d \vartheta
\end{aligned}
$$

Comparison with (A5) given

$$
\Phi^{\prime}(\vartheta)=\frac{i \cos \vartheta}{4 \pi \rho_{0} \alpha_{0}^{2}}
$$

and

$$
\Phi(9)=\frac{i \cos \vartheta \varepsilon^{1 / 2}}{4 \pi \rho_{0}^{1 / 2} \alpha_{0}^{3 / 2}}
$$

where $\Phi(\vartheta)$ is the approximate weight for the Gaussian beam representation of $P$ wave radiation from a two-dimensional point force and $\vartheta$ is the angle between the direction of the point force and the beam direction.

Acknowledgments. The authors wish to thank V. Cerveny' and I. PŠenčik for preprints, lecture notes, and the use of their ray tracing program, RAY81. Thanks are also given to $M$. Fehler for supplying data from the Mount Saint Helens seismic study, and to V. Cormier and R. Madariaga for informative discussions. Most of the computation in example I was done by one of the authors (K.A.) at the Geophysical Institute of the University of Alaska while he was working there as the distinguished visiting professor. The computational assistance given by N. Biswas and J. F. Lico is gratefully acknowledged. This work was supported by the Defense Advanced Research Projects Agency under grant F49620-82-K-0004 monitored by the Air Force Office of Scientific Research and by Schlumberger-Doll research funds.

\section{REFERENCES}

Aki, K., Scaltering of $P$ waves under the Montana LASA, J. Geophys. Res., 78, 1334-1346, 1973.

Aki, K., and P. G. Richards, Quantitative Seismology: Theory and Methods, W. H. Freeman, San Francisco, Calif., 1980

Azbel, 1. Y., L. A. Dmitrieva, and T. B. Yanovskaya, A method of calculating geometrical divergence for a medium inhomogeneous in three dimensions, Comput. Seismol., 13, 113-127, 1980.

Babich, V. M., and M. M. Popov, Propagation of concentrated sound beams in a three-dimensional inhomogeneous medium, Sov. Phys. Acoust., 27, 459-462, 1981.

Bard, P.-Y., and M. Bouchon, The seismic response of sediment-filled valleys, 1, The case of incident $S H$ waves, Bull. Seismol. Soc. Am. 70, 1263-1286, 1980.

Boore, D. M., K. L. Larner, and K. Aki, Comparison of two independent methods for the solution of wave-scattering problems: Response of a sedimentary basin to vertically incident $S H$ waves, $J$. Geophys Res., 76, 558-569, 1971.

Capon, J., Characterization of crust and upper mantle structure under LASA as a random medium, Bull. Seismol. Soc. Am., 64, 235-266, 1974.

Cerveny, V., Expansion of a plane wave into Gaussian beams, Stud. Geophys. Geod., 26, 120-131, 1982.

Cervený, V., Synthetic body wave seismograms for laterally varying layered structures by the Gaussian beam method, Geophys. $J$. R. Astron. Soc., 73, 389-426, 1983.

Cervený, V., and F. Hron, The ray series method and dynamic ray tracing system for three-dimensional inhomogeneous media., Bull. Seismol. Soc. Am., 70, 47-77, 1980.

Cervený, V., and I. Pšenčik, Ray amplitudes of seismic body waves in laterally inhomogeneous media, Geophys. J. R. Astron. Soc., 57, 91-106, 1979.

Červený, V., and I Pšenčik, Gaussian beams and paraxial ray approximations in three-dimensional elastic inhomogeneous media, $J$. Geophys., 53, 1-15, 1983a.

Cervený, V., and I. Pšenčík, Gaussian beams in two-dimensional elastic inhomogeneous media, Geophys. J. R. Astron. Soc., 72, 417-433, $1983 b$.

Červený, V., I. A. Molotkov, and I. Pšenčík, Ray Method in Seismology, Karlova Univerzita, Prague, 1977.

Črvený, V., M. M. Popov, and I. Pšenčík, Computation of wave fields in inhomogeneous media-Gaussian beam approach, Geophys. J. R. Astron. Soc., 70, 109-128, 1982.

Chapman, C. H., A new method for computing synthetic seismograms, Geophys. J. R. Astron. Soc., 54, 481-513, 1978.

Chapman, C. H., and R. Drummond, Body-wave seismograms in 
inhomogeneous media using Maslov asymptotic theory, Bull. Seismol. Soc. Am., 72, s277-s317, 1982.

Claerbout, J. F., Fundamentals of Geophysical Data Processing, McGraw-Hill, New York, 1976.

Cline, A. K., FITPACK - A software package for curve and surface fitting employing splines under tension, Dep. of Comput. Sci., Univ. of Tex., Austin, 1981

De Boor, C., A Practical Guide to Splines, Springer-Verlag, New York, 1980.

Fehler, M., and B. Chouet, Operation of a digital seismic network on Mount St. Helens volcano and observations of long period seismic events that originate under the volcano, Geophys. Res. Lett., 9, 1017-1020, 1982.

Gloge, D., and D. Marcuse, Formal quantum theory of light rays, $J$ Optic. Soc. Am., 59, 1629-1631, 1969.

Hayakawa, M., Prospecting of the underground structure of "Showashinzan" by various geophysical methods, particularly seismic survey, Jpn J. Geophys., 1, 13-20, 1957.

Hong, T. L., and D. V. Helmberger, Glorified optics and wave propar gation in non-planar structures, Bull. Seismol. Soc. Am., 68, 13131330, 1977.

Kienle, J., D. J. Lalla, C. F. Pearson, and S. A. Barrett, Search for shallow magma accumulation at Augustine volcano, contract AT(45-1)-2229, U.S. Dep. of Energy, Washington, D. C., 1979.

Kirpichnikova, N. J., Construction of solutions concentrated close to rays for the equations of elasticity theory in an inhomogeneous isotropic space (in Russian.), in Mathematical Problems of Theory of Diffraction and Propagation of Waves, vol. 1, Nauka, Leningrad, 1971. (English translation, Proc. Steklov Inst. Math., 115, 114-126, 1974.)

Kjartansson, E., Attenuation of seismic waves in rocks and applications in energy axploration, Ph.D. thesis, Stanford Univ., Stanford, Calif., 1980.

Kravtsov, Y. A., Two new asymptotic methods in the theory of waves in laterally inhomogeneous media, Sov. Phys. Acoust., 14, 1-17, 1968.

Kravtsov, Y. A., and Y. I. Orlov, Limits of applicability of the method of geometric optics and related problems, Sov. Phys. Usp., 23, 750 762,1980 ,
Lalla, D. J., and J. Kienle, Comparison of synthetic and observed seismograms for volcanic earthquakes at Augustine volcano (abstract, paper presented at Pacific Northwest Regional Meeting. AGU, Washington, D. C., 1982.

Marcuse, D., Light Transmission Opties, Van Nostrand, New York, 1982.

Marcuvitz, N., Quasi-particle view of wave propagation, Proc. IEEE, $68,1380-1395,1980$.

Popov, M. M., A new method of computation of wave fields in highfrequency approximation (in Russian), in Mathematical Problems of Theory of Propagation of Waves, vol. 11, pp. 195-216, Nauka, Leningrad, 1981. (English trạnslation, J. Sov. Math., 2O(1), 1869-1882, 1982.)

Popov, M. M., A new method of computation of wave fields using Gaussian beams, Wave Motion, 4, 85-97, 1982.

Popov, M. M., and I. Pšenčík, Ray amplitudes in inhomogeneous media with curved interfaces, Geofyzs. Sb., 24, 118-129, 1976.

Popov, M. M., and I. Pšenčik, Computation of ray amplitudes in inhomogeneous media with curved interfaces, Stud. Geophys. Geod. 22, 248-258, 1978.

Pretlova, V., Bicubic spline smoothing of two-dimensonal geophysical data, Stud. Geophys. Geod., 20, 168-177, 1976.

Pšenčík, I., Program for the computation of kinematic and dynamic parameters of seismic body waves in 2-D laterally inhomogeneous media with curved interfaces (in Russian), in Programs for the Interpretation of Seismic Observations, vol. 3, Nauka, Leningrad, 1983.

Tappert, F. D., The parabolic approximation method, Wave Propagation and Underwater Acoustics, Lect. Notes Phys., 70, 224-287, 1976

K. Aki and R. Nowack, Department of Earth, Atmospheric, and Planetary Sciences, Massachusetts Institute of Technology, Cambridge, MA 02139 .

(Received July 7, 1983 revised February 29, 1984 accepted March 19, 1984.) 